\title{
The yeast telomerase RNA, TLC1, participates in two distinct modes of TLC1-TLC1 association processes in vivo
}

Tet Matsuguchi, Elizabeth Blackburn

Telomerase core enzyme minimally consists of the telomerase reverse transcriptase domain-containing protein (Est2 in budding yeast S. cerevisiae) and telomerase RNA, which contains the template specifying the telomeric repeat sequence synthesized. Here we report that in vivo, a fraction of S. cerevisiae telomerase RNA (TLC1) molecules form complexes containing at least two molecules of TLC1, via two separable modes: one requiring a sequence in the $3^{\prime}$ region of the immature TLC1 precursor and the other requiring $\mathrm{Ku}$ and Sir4. Such physical TLC1-TLC1 association peaked in G1 phase and did not require telomere silencing, telomere tethering to the nuclear periphery, telomerase holoenzyme assembly, or detectable Est2-Est2 protein association. These data indicate that TLC1-TLC1 associations reflect processes occurring during telomerase biogenesis; we propose that TLC1-TLC1 associations and subsequent reorganization may be regulatory steps in telomerase enzymatic activation. 
1 The Yeast Telomerase RNA, TLC1, Participates in Two Distinct Modes of TLC1-TLC1

2 Association Processes in vivo

3

4 ABSTRACT

5 Telomerase core enzyme minimally consists of the telomerase reverse transcriptase domain-

6 containing protein (Est2 in budding yeast $S$. cerevisiae) and telomerase RNA, which contains the

7 template specifying the telomeric repeat sequence synthesized. Here we report that in vivo, a

8 fraction of S. cerevisiae telomerase RNA (TLC1) molecules form complexes containing at least

9 two molecules of TLC1, via two separable modes: one requiring a sequence in the 3' region of

10 the immature TLC1 precursor and the other requiring Ku and Sir4. Such physical TLC1-TLC1

11 association peaked in G1 phase and did not require telomere silencing, telomere tethering to the

12 nuclear periphery, telomerase holoenzyme assembly, or detectable Est2-Est2 protein association.

13 These data indicate that TLC1-TLC1 associations reflect processes occurring during telomerase

14 biogenesis; we propose that TLC1-TLC1 associations and subsequent reorganization may be

15 regulatory steps in telomerase enzymatic activation.

17 AUTHORS

18 Tet Matsuguchi

19 Elizabeth H. Blackburn

20

21 Department of Biophysics and Biochemistry

22 University of California, San Francisco

23 San Francisco, CA

24 United States of America

25

26 Corresponding Author

27 Elizabeth H. Blackburn

28600 16th Street Box 2200

29 San Francisco, CA 94158-2517

$30 \quad 415-476-2824$

31 Elizabeth.Blackburn@ucsf.edu 
32

33

34

35

36

37

38

39

\section{INTRODUCTION}

Telomeric DNA is typically composed of repetitive sequences (TG1-3 repeats in the budding yeast $S$. cerevisiae) that allow recruitment of specialized macromolecular complexes that help replenish and protect telomeres (de Lange, Lundblad \& Blackburn, 2006). These include the ribonucleoprotein telomerase, which adds telomeric DNA by the action of its reverse transcriptase-containing subunit (Est2 in $S$. cerevisiae), templated by a sequence within the telomerase RNA component (TLC1 in S. cerevisiae), as well as telomere-protective doublestranded and single-stranded telomeric DNA binding proteins, such as Rap1 and Cdc13 in yeast (Jain \& Cooper, 2010).

Budding yeast telomerase RNA, TLC1, is over 1300 nucleotides in size and, in addition to providing the template for reverse transcription, has extensive secondary structures (Zappulla $\&$ Cech, 2004). Certain structures within TLC1 have been defined and form binding sites for Est2 and other telomerase factors. The critical central core of TLC1 includes a structurally highly conserved pseudoknot to which Est2 binds, while an Sm-protein binding site is located near the 3' end, which is important for the stability and processing of immature TLC1 (Seto et al., 2002; Zappulla \& Cech, 2004; Lin et al., 2004; Jiang et al., 2013) (Figure 1A). Previously, it was reported that mutations ( $t l c l-42 G$ and $t l c 1-42 C$ ) in a 6-base palindromic sequence, located within the TLC1 precursor 3' region that is cleaved off to form the processed mature TLC1 RNA (see Figure 1A), cause telomeres to be shorter in vivo and abrogate dimerization of TLC1 precursor synthesized in vitro (Gipson et al., 2007). Additionally, a 48-nucleotide stem motif in TLC1 directly binds the Ku70/Ku80 complex, which, in addition to its widely conserved canonical role in non-homologous end joining (NHEJ), is required for many aspects of yeast telomere biology (Stellwagen et al., 2003). This TLC1-Ku interaction, while not absolutely required for telomere maintenance by telomerase in vivo, is required for maintenance of full-length telomeres, in vivo association of Est2 to telomeres in G1 phase of the cell cycle (Fisher, Taggart \& Zakian, 2004), full recruitment of telomeres to the nuclear periphery (Taddei et al., 2004), and transcriptional silencing at telomeres (Boulton \& Jackson, 1998). A mutant $\mathrm{Ku}$ containing a small insertion, $y k u 80-135 i$, specifically abrogates the TLC1-Ku interaction but leaves NHEJ intact (Stellwagen et al., 2003). Est1 and Est3 are essential factors for telomerase, which together with Est2 and 
63 TLC1, make up the telomerase holoenzyme. Est1 associates with the telomerase complex by 64 directly binding to a bulge-stem region of TLC1 conserved in several budding yeasts, and this

65

67 association is critical for the recruitment of telomerase to telomeres (Seto et al., 2002; Chan, Boulé \& Zakian, 2008).

Human, S. cerevisiae, and Tetrahymena (ciliated protozoan) telomerases have been inferred to be active as a monomer in vitro (Bryan, Goodrich \& Cech, 2003; Alves et al., 2008; Shcherbakova et al., 2009; Jiang et al., 2013). However, reports have also suggested that the human, $S$. cerevisiae, and Euplotes (ciliated protozoan) telomerase complexes can exist in a dimeric (or other oligomeric) forms (Prescott \& Blackburn, 1997; Wenz et al., 2001; Beattie et al., 2001; Wang, Dean \& Shippen, 2002). Recent single-molecule electron microscopic structural determinations indicate that core human telomerase complex (telomerase RNA, hTER, and reverse transcriptase, hTERT) is a dimer in vitro held together by RNA-RNA (hTER-hTER) interaction (Sauerwald et al., 2013).

Here, we explored possible modes of physical telomerase dimerization in vivo, focusing on the yeast telomerase RNA component TLC1. We developed a biochemical method that directly demonstrates a physical TLC1-TLC1 association (dimerization/oligomerization; direct or indirect), quantified in extracts of cells expressing normal amounts of telomerase RNA from the endogenous TLC1 gene chromosomal locus. We have not determined whether there are more than two molecules of TLC1 that are associated in complexes, so for simplicity, we refer to this as TLC1-TLC1 association. We report here that such TLC1-TLC1 associations occur in vivo via two modes, each mode having distinctive requirements. Our evidence supports association between telomerase RNAs occurring during the biogenesis of active telomerase complex, with potential functional importance in the regulation of telomerase activity.

\section{MATERIALS AND METHODS}

\section{$\underline{\text { Plasmids }}$}

The integrating vector, pRS306-TLC1, was provided by Jue Lin. The MS2 CP-binding RNA hairpins were constructed by annealing overlapping oligonucleotide in a standard PCR protocol. 
94 The hairpin construct was cloned into the BclI site of pRS306-TLC1. The fusion PCR method 95 was used to construct $t l c 1-42 G$ and $t l c 1-42 C$ alleles, which were cloned between the BclI and 96 XhoI sites of pRS306-TLC1. CEN-ARS versions of the plasmids were made by subcloning 97 BamHI-XhoI fragments of the integrating vectors into the vector pRS316.

99 Yeast strains and growth media

100 Yeast strains were in the S288c background and are isogenic with BY4746, except as noted in

101 Table 1 (Baker Brachmann et al., 1998; Tomlin et al., 2001). Yeast cultures were grown in

102 standard rich medium or minimal media (YEPD or CSM). Deletion strains were made using a

103 PCR-based transformation method (Longtine et al. 1998). Cell cycle synchronization was

104 achieved by addition of $5 \mathrm{uM}$ alpha-factor for 4 hours, which arrests yeast cells in G1 phase. The

105 cells were released from the arrest by washing away media containing alpha-factor and addition

106 of new media.

107

Immunoprecipitation of MS2 hairpin-tagged TLC1

109 TLC1 was tagged with two MS2 coat-protein-binding RNA hairpins at the BclI restriction site in 110 the TLC1 coding region sequence. This gene construct with its native promoter was integrated at 111 the endogenous chromosomal TLC1 locus, in tandem with untagged, wild-type TLC1, flanking

112 the URA3 marker. MS2 coat protein fused to 3 Myc epitope tags was expressed either in $t l c 1 \Delta$ or 113 in experimental strains containing both tagged and untagged TLC1. The MS2 coat protein from

114 tlc1 $\Delta$ strains were used for coIP experiments using strains yEHB22,807-824. Whole cell lysates

115 were prepared from cultures in log-phase of growth in YEPD $\left(\mathrm{OD}_{600}=0.6-1.0\right)$ using glass beads

116 and bead beaters. The lysis/wash buffer contained 50mM HEPES-KCl pH8.0, 2 mM EDTA, 2

117 mM EGTA, 0.1\% Nonidet P40, 10\% glycerol, cOmplete EDTA-free protease inhibitors (Roche)

118 and $\mathrm{RNasin}$ (Promega; $1 \mathrm{uL} / \mathrm{mL}$ ). The lysate concentrations were adjusted to $\mathrm{A}_{260 \mathrm{~nm}}=40$ before

119 immunoprecipitation. For lysates containing co-expressed MS2 coat protein, $400 \mathrm{uL}$ of lysate

120 was mixed with $1.5 \mathrm{mg}$ Dynal ProA magnetic beads (Invitrogen) and $1 \mathrm{ug}$ of monoclonal anti-

121 Myc antibody (9E11, Santa Cruz Biotechnology). For experiments in which MS2 coat protein

122 was purified separately, ProA magnetic beads, anti-Myc antibody, and whole cell lysate

123 containing MS2 coat protein (at $\left.A_{260 n m}=60-80\right)$ were incubated for 1-2 hours. The

124 immunoprecipitation was allowed to take place at $4{ }^{\circ} \mathrm{C}$ for 4 -hours to overnight. The 
125 immunoprecipitates were washed 3 times with the wash buffer. For oligonucleotide-directed

126 displacement experiments, the immunoprecipitates on the beads were aliquotted in separate tubes

127 after the first wash. Each sample was subjected to different sets of oligonucleotides diluted to 0.5

$128 \mathrm{uM}$ each in the wash buffer and incubated for 1 hour. Further washes were carried out as above

129 before RNA extraction.

130

131 Immunoprecipitation of tagged proteins

132 For immunoprecipitation of tagged proteins (Est2-13xMyc, Est2-3xFLAG), lysates were

133 prepared as described above. For Myc-tagged proteins, the lysate was mixed with $1.5 \mathrm{mg}$ Dynal

134 ProA magnetic beads, and 1 ug 9E11 antibody. For FLAG-tagged proteins, lysate was incubated

135 with $50 \mathrm{uL}$ of M2-conjugated agarose beads. For sequential immunoprecipitation of FLAG-

136 tagged proteins followed by Myc-tagged proteins, $15 \mathrm{ug}$ of 3xFLAG peptide was added to the

137 M2-conjugated agarose beads. The eluate was then used for Myc-tag immunoprecipitation as

138 described.

139

140 Quantitative reverse transcription and PCR (qRT-PCR)

141 RNA from input and immunoprecipitates were isolated using RNeasy Mini Kit (Qiagen),

142 including the DNase step as described by the manufacturer. The primer set for PGK1 was

143 designed using IDT's PrimerQuest program. The reverse primers used to distinguish tagged and

144 untagged TLC1 were designed within and at the insertion junction, respectively, of the MS2

145 hairpin tag. One-step reverse transcription and PCR kits were used for all RNA quantifications,

146 except for the quantification of immature TLC1 (Stratagene, Invitrogen). For quantification of

147 immature TLC1, or 3' regions of TLC1, SuperScript III and random hexamer were used for

148 reverse transcription. Subsequently, SYBR Green I Master mix kit (Roche) was used for

149 quantitative PCR. All quantitative PCR runs included serially diluted RNA samples to make

150 standard curve, from which relative quantitative values were derived using the LightCycler

151 software. The oligonucleotide sequences used in qRT-PCR reactions are listed in Table 2.

152

153 Calculation of Fraction TLC1 in Dimer Form

154 Four quantitative values from qRT-PCR assays are used to estimate the fraction of TLC1 in

155 dimer form: untagged and MS2-tagged TLC1 in the input lysate $\left(\mathrm{TLC}_{\mathrm{IN}}, \mathrm{MS} 2_{\mathrm{IN}}\right)$, untagged and 
156 MS2-tagged TLC1 in MS2-immunoprecipitate (TLC1 $\left.1_{\mathrm{IP}}, \mathrm{MS}_{\mathrm{IP}}\right)$. The following equations are 157 used:

(1) $\mathrm{f}(\mathrm{TLC} 1)=\mathrm{TLC} 1_{\mathrm{IN}} /\left(\mathrm{TLC} 1_{\mathrm{IN}}+\mathrm{MS} 2_{\mathrm{IN}}\right)$

159

(2) $\mathrm{f}(\mathrm{IP})=\mathrm{MS} 2_{\mathrm{IP}} / \mathrm{MS}_{\mathrm{IN}}$

160

(3) $\mathrm{n}($ heterodimer $)=\mathrm{TLC}_{\mathrm{IP}} / \mathrm{f}(\mathrm{IP})$

161

(4) $\mathrm{f}$ (heterodimer $)=2 * \mathrm{f}(\mathrm{TLC} 1) *(1-\mathrm{f}(\mathrm{TLC} 1))$

162

(5) $\mathrm{n}($ dimer $)=\mathrm{n}($ heterodimer $) / \mathrm{f}$ (heterodimer $)$

(6) $\mathrm{n}(\mathrm{TLC} 1$ in dimer $)=2 * \mathrm{n}$ (dimer)

(7) $\mathrm{f}($ dimer $)=\mathrm{n}(\mathrm{TLC} 1$ in dimer $) /\left(\mathrm{TLC1}_{\mathrm{IN}}+\mathrm{MS}_{\mathrm{IN}}\right)$

In the equations above $f()$ represents "fraction of" and $n()$ represents "amount of." (1) Fraction of TLC1 RNAs that are untagged is calculated as the fraction untagged divided by the sum of untagged and MS2-tagged RNAs. (2) Fraction of MS2-tagged TLC1 immunoprecipitated is calculated by dividing the amount of MS2-tagged TLC1 in the precipitate by the amount of MS2-tagged TLC1 in the input lysate. (3) The amount of untagged TLC1 in the precipitate represents untagged TLC1 in the heterodimeric form with the MS2-tagged TLC1. The total amount of heterodimeric TLC1 is estimated by dividing the amount of untagged TLC1 in the precipitate by the fraction of MS2-tagged TLC1 precipitated (Equation 2). (4) The fraction of TLC1 dimers that are in homodimeric (untagged+untagged or MS2-tagged+MS2-tagged) and heterodimeric (untagged+MS2-tagged) are assumed to result from random associations $\left(\mathrm{f}(\mathrm{TLC} 1)^{2}, \mathrm{f}(\mathrm{MS} 2)^{2}, 2 * \mathrm{f}(\mathrm{TLC} 1) * \mathrm{f}(\mathrm{MS} 2)\right)$. Therefore, the fraction of TLC1 dimers in the heterodimeric form is calculated as $2 * \mathrm{f}(\mathrm{TLC} 1) * \mathrm{f}(\mathrm{MS} 2)$ or $2 * \mathrm{f}(\mathrm{TLC} 1) *(1-\mathrm{f}(\mathrm{TLC} 1)) .(5)$ The total amount of dimer is calculated by dividing the number of heterodimers (Equation 3) by the fraction of dimers that are heterodimeric (Equation 4). (6) The total amount of TLC1 in calculated by doubling the amount of dimer (Equation 5). (7) Fraction of TLC1 in dimer form is calculated by dividing the amount of TLC1 in dimer form (Equation 6) by the total amount of

Telomere length analysis

184 Genomic DNA was digested with XhoI and separated on a $0.85 \%$ agarose gel. DNA was 185 denatured and transferred to a Nylon membrane, and UV-crosslinked with a Stratalinker. The 186 membrane was blotted with telomeric oligonucleotide 
187 (5'-CACACCCACACCACACCCACAC-3') labeled with WellRED D3 fluorescent dye at the 5'

188 end. The blotted membrane was scanned and analyzed using the Odyssey Infrared Imaging

189 System (LI-COR). A linear plasmid containing an S. cerevisiae telomeric DNA sequence was

190 included as a marker of size $\sim 500 \mathrm{bp}$.

191

192

193

RESULTS

194

Co-immunoprecipitation assays demonstrate TLC1-TLC1 association in vivo

195

196 To quantify the association between different TLC1 molecules in yeast whole-cell extracts, a co-

197 immunoprecipitation (coIP) assay was developed. First, we created a tagged TLC1 RNA for

198 immunoprecipitation using a tandem pair of RNA hairpins that specifically bind to the

199 bacteriophage MS2 Coat Protein. This two-hairpin construct was inserted at one of two sites in

200 regions of TLC1 previously shown to accommodate insertions of modular protein binding

201 domains with minimal if any effect on in vivo functions (Bernardi \& Spahr, 1972; Zappulla \&

202 Cech, 2004) (Figure 1A). Secondly, we fused three copies of myc tag to MS2 Coat Protein and

203 integrated this gene construct into the genome of experimental strains. Co-expression of the MS2

204 hairpin-tagged TLC1 (TLC1-MS2) and myc-tagged Coat Protein (CP-3myc) allowed specific

205 immunoprecipitation of TLC1-MS2 using an anti-myc antibody. Thirdly, we developed

206 quantitative RT-PCR assays to measure levels and recovery of TLC1, using two sets of PCR

207 primers capable of distinguishing and specifically amplifying either the untagged TLC1 or

208 TLC1-MS2 (Figure 2A and 2B).

209 Next, we verified that the insertion of the MS2 tag did not significantly alter TLC1 functions in

210 vivo. We tested the telomere lengths of strains that have MS2 hairpin insertion at two different

211 sites, nucleotide positions 455 and 1033 of TLC1 (NcoI and Bcll sites). The insertion had the

212 least impact at nucleotide position 1033 (Figure 1C), and we used this construct for the rest of

213 the study. The expression level of TLC1-MS2 was comparable to untagged TLC1 (Figure 2C).

214 The association of TLC1-MS2 with Est2 was slightly reduced compared to untagged TLC1,

215 consistent with telomere lengths observed in cells expressing only TLC1-MS2 (Figure 2D). 
217 Finally, we co-expressed TLC1-MS2 and untagged TLC1 from the endogenous TLC1 locus to

218 test the coIP of untagged TLC1 with TLC1-MS2. As a control, an equal number of cells from

219 two independently cultured strains expressing either only untagged TLC1 or only TLC1-MS2

220 were mixed prior to cell lysis ("Mix" samples in figures). We found that 50-80\% of total TLC1-

221 MS2 is immunoprecipitated from lysates made from the co-expression strain and from the mixed

222 population. A significant enrichment of untagged TLC1 in the TLC1-MS2 immunoprecipitate

223 was observed only in the co-expression strain and not in the mixed cell population, indicating

224 that this assay detected bona fide in vivo association of separate TLC1 molecules (see Materials

225 and Methods and Figure 2E). After adjusting for the immunoprecipitation efficiency and the fact

226 that this coIP assay only detects heterodimer of TLC1-MS2 and untagged TLC1, we determined

227 that in unsynchronized log phase cell populations, at least $10 \%$ of TLC1 is associated with

228 another TLC1 in vivo (Figure 2E; see Materials and Methods for calculation). Interestingly, we

229 observed that the fraction of immature TLC1 molecules present in the whole lysate (4-8\%)

230 (Mozdy \& Cech, 2006) did not significantly change in the immunoprecipitate, indicating that

231 both immature and mature forms of TLC1 participate comparably in TLC1-TLC1 association

232 (Figure 2F).

233

234 The 3' Region of TLC1 is Important for TLC1-TLC1 association

235

236 To determine the regions of TLC1 involved in the TLC1-TLC1 physical association, we

237 designed a nucleic acid competition experiment aimed to disrupt this association by incubating

238 the TLC1 complex(es), extracted as the immunoprecipitates from cell lysates, with excess anti-

239 sense oligonucleotides. We designed 72 overlapping DNA oligonucleotides, each 30 bases in

240 length, that in total were complementary to the full length of the immature TLC1, which includes

241 the 3' region that is cleaved off in the mature form (Figure 1B). These oligonucleotides were

242 incubated with the TLC1-MS2 immunoprecipitate bound to the magnetic beads in the wash

243 buffer (see Materials and Methods). We predicted that the collection of these 72 TLC1 antisense

244 oligos would act as competitors to TLC1-TLC1 association in the immunoprecipitates. As a

245 control, 72 different DNA oligonucleotides designed against other regions of the yeast genome

246 were used. Incubation of the full set of 72 TLC1-antisense oligonucleotides (but not the 72

247 control oligonucleotides) with the immunoprecipitates reduced the amount of untagged TLC1 
248 remaining on the affinity beads by about $70 \%$, while not appreciably diminishing the amount of

249 TLC1-MS2 remaining bound to the affinity beads (Figure 3A and B, bottom row). This result

250 indicated that the 72 TLC1-antisense oligonucleotides likely disrupted the association of the

251 untagged TLC1 and TLC1-MS2.

252

253 To further delineate the regions important for the TLC1-TLC1 association, different subsets of

254 oligonucleotides were used in the same experimental set-up. The 72 oligonucleotides were

255 subdivided into intervals encompassing thirds or ninths of the length of the immature TLC1, in

256 order to probe each TLC1 region separately (Figure 3B). The oligonucleotides complementary to

257 the first third (the 5' region) of TLC1 had little effect on disrupting TLC1-TLC1 association,

258 while the oligonucleotides against the central and 3' region intervals had greater effects (Figure

259 3B, Row 2). Even added together, the total of the effects from each of the three separate regions

260 was significantly less than the disruptive effect seen when all 72 oligonucleotides were added

261 simultaneously, suggesting that there is a synergistic effect in adding all oligonucleotides at once.

262 Similarly, separately challenging the TLC1-TLC1 immunoprecipitates in this way with the anti-

263 sense oligonucleotides encompassing each of the one-ninth regions, especially in the 5' regions

264 of TLC1, disrupted the TLC1-TLC1 association to even lesser extents (Figure 3B Row 1).

265

266 Interestingly, TLC1-TLC1 association was disrupted by $30 \%$ using the eight antisense

267 oligonucleotides encompassing the TLC1 3' region. Only two of these eight oligonucleotides

268 were complementary to the last 21 bases of the mature form of TLC1; the remaining six

269 oligonucleotides were complementary only to the 3' extension of the un-cleaved, immature form

270 of TLC1 (Figure 1B). As described above, the immature TLC1 molecules accounted for only 4-

$2718 \%$ of the total TLC1 signal in the immunoprecipitate (Figure 2F); thus, a reduction solely of

272 immature TLC1 precursors cannot account for the $30 \%$ disruption by the 3 ' most one-ninth

273 TLC1-complementary oligonucleotides. This result suggests that a small region (30 bases)

274 encompassed by just two oligonucleotides had a relatively large effect in disrupting TLC1-TLC1

275 association of the mature form of TLC1.

276

277 Together, these findings indicated that the 3' region of TLC1 transcript is either the most critical

278 for TLC1-TLC1 association to occur in vivo, and/or the most vulnerable to subsequent in vitro 
279 disruption of the associated form. This in vitro disruption by the 3' region-targeting

280 oligonucleotides could have been through a direct competition of base-paired regions between

281 two TLC1 RNAs, through an unwinding of some structural elements of TLC1, or disruption of

282 RNA-protein associations. Additionally, these data suggest that the TLC1-TLC1 association

283 mostly involves tail-tail (i.e., 3'region with 3' region) interactions, rather than head-head (i.e.,

284 5'region with 5' region) or head-tail (i.e., 5'region with 3' region) formations.

285

286 Prompted by the importance of the 3' region of TLC1, we tested the potential role in TLC1-

287 TLC1 association for a previously identified, palindromic sequence located in the 3' region

288 cleaved off during TLC1 maturation and thus present only in the immature, precursor TLC1

289 molecules. This palindromic sequence is evolutionarily conserved among budding yeast species

290 (Gipson et al., 2007). Two palindrome disruption mutations (tlcl-42G and tlcl-42C) that prevent

291 potential intermolecular base-pairing by this sequence, and the compensatory mutations (tlcl-

$29242 G$ and tlcl-42C in trans), which restore the potential for intermolecular base-pairing but not

293 the wild-type palindromic sequence itself, have been described previously (Gipson et al., 2007).

294 We found that the palindrome disruption mutations $t l c 1-42 G$ and $t l c 1-42 C$, when incorporated

295 into untagged TLC1 in the strains also expressing TLC1-MS2, reduced TLC1-TLC1 coIP by

296 over half (Figure 3C). The compensatory mutations, tlc1-42G and tlc1-42C in trans, although

297 restoring intermolecular base-pairing potential, failed to restore the TLC1-TLC1 coIP level

298 (Figure 3C). The total levels of these mutant telomerase RNAs were unchanged from wild type;

299 hence, efficient in vivo association between mature TLC1 molecules requires the specific

300 sequence - and not simply its potential for base pairing in trans - of a palindromic motif located

301 in the cleaved-off 3' portion of the TLC1 precursor. These results indicate that at least some

302 TLC1-TLC1 association initiates during telomerase biogenesis before processing produces the

303 mature TLC1 3' end.

304

305 TLC1-TLC1 Association is dependent on nuclear export and is cell cycle-regulated

306 Maturation of telomerase RNA including 3' end processing takes place partially in the cytoplasm

307 (Gallardo et al., 2008). Interestingly, while deletion of Tgs1, which is responsible for TLC1 m3G

308 cap formation (Franke, Gehlen \& Ehrenhofer-Murray, 2008), had no effect on total TLC1 levels 
309 and little effect on TLC1-TLC1 association ( $\mathrm{p}>0.05)$, mutating Nup133 (required for nuclear

310 export) (Gallardo et al., 2008) diminished by at least half the fraction of TLC1 in the associated

311 form, while causing no effect on total TLC1 levels $(\mathrm{p}<0.05$; Figure 4A). This finding indicated

312 that TLC1 export into the cytoplasm may be necessary for TLC1-TLC1 association.

313 TLC1 maturation by 3' end processing is reported to be active only during G1 phase of the cell

314 cycle (Chapon, Cech \& Zaug, 1997). To test whether TLC1-TLC1 association is controlled

315 during the cell cycle, yeast cell lysates were prepared at 15-minute intervals from cells following

316 release into G1 phase from an alpha-factor arrest. Cell cycle progression and synchrony were

317 confirmed by analysis of the various cyclin mRNA levels throughout the time course (Figure

318 4B). Consistent with a previous report (Mozdy \& Cech, 2006), the total TLC1 steady-state levels

319 showed a slight increase as the cell cycle progressed (Figure 4C). During the first cell cycle after

320 the release from the 2-hour alpha-factor arrest, the fraction of TLC1 in dimer form in the coIP

321 assay remained relatively constant (Figure 4D). Then after mitosis, as the cell population re-

322 entered the next G1 phase, the fraction of TLC1-TLC1 association abruptly increased 2-fold,

323 with markedly different kinetics compared to the slow and steady accumulation of total

324 TLC1 throughout the cell cycle progression (Figure 4D). This finding is consistent with TLC1-

325 TLC1 association occurring during the biogenesis of telomerase complex, a process that has been

326 detected only in G1 phase. The lack of a higher fraction of TLC1 in the dimer form during the

327 G1 phase immediately following the release from the 2-hour alpha-factor arrest is also consistent

328 with TLC1-TLC1 association during a biogenesis step, since in this situation, cells have been

329 held in G1 phase, in the presence of active biogenesis machinery, for 120 minutes prior to the

330 point of release from alpha-factor. We conclude that TLC1-TLC1 association is cell-cycle-

331 controlled and highest in G1.

332 Telomerase holoenzyme formation is not required for TLC1-TLC1 association

333

334 To test whether there are any protein factors that assist in maintaining the TLC1-TLC1

335 association, we treated the immunoprecipitates with trypsin. We found that protease treatment

336 reduced coIP efficiency by $\sim 40 \%$ compared with the control (Figure 5A), suggesting a role for

337 protein(s) in initiating, or stabilizing, TLC1-TLC1 association.

338 
339 We tested the most likely protein factor candidate, Est2, the telomerase reverse transcriptase core

340 protein. It has been shown that Est2 and TLC1 come together in the cytoplasm, although when in

341 the cell cycle they initiate the interaction is unclear (Teixeira et al., 2002; Gallardo et al., 2008).

342 In est $2 \Delta$ strains, a diminution in TLC1-TLC1 association of about $20-25 \%$ was detected,

343 although this measured reduction was not highly significant when compared to the control wild-

344 type EST2 strain ( $>>0.05$; Figure 5B). We reasoned that the modest requirement for Est 2 in

345 TLC1-TLC1 association might be reflected in TLC1 mutants known to disrupt the core

346 pseudoknot structure required for Est2-TLC1 interaction. Therefore, we disrupted the TLC1

347 pseudoknot by mutating either side of one stem (intra-base-pairing) made up of conserved

348 sequences CS3 and CS4 (tlcl-20 and tlc1-21), and restored the pseudoknot structure by the

349 compensatory mutations (tlc1-22) (Lin et al., 2004). CoIP assays showed that the in vivo TLC1-

350 TLC1 association was substantially reduced by the pseudoknot-disruptive mutations tlc1-20 and

$351 t l c 1-21$ and fully restored by the compensatory mutations, tlc1-22 (Figure 5C). Thus, efficient

352 TLC1-TLC1 association requires at least this aspect of normal folding of TLC1, although

353 binding to Est2 is largely dispensable.

354

355 Next, we tested two other essential components of the telomerase holoenzyme, Est1 and Est3, for 356 any roles in the in vivo TLC1-TLC1 association. Est1-TLC1 interaction is limited to S-phase of

357 the cell cycle, and Est3 interaction with Est2 requires Est1 and hence is also S-phase dependent

358 (Osterhage, Talley \& Friedman, 2006). As in the est $2 \Delta$ strain, the est $3 \Delta$ strain showed a modest

359 but not significant ( $\mathrm{p}>0.05$, Figure 5B) reduction in TLC1-TLC1 association. In est1 $\Delta$, however,

360 the TLC1-TLC1 association was reduced by $\sim 35 \%(\mathrm{p}<0.05$, Figure 5B). While many aspects of

361 Est1 functions in telomere biology remain unclear, roles for Est1 in the recruitment of telomerase

362 to telomeres as well as in telomerase enzymatic activation are well established (Evans \&

363 Lundblad, 2002). The TLC1-TLC1 association showed a somewhat greater dependence on Est1

364 than on Est2 and Est3. This raises the possibility that, rather than the telomerase enzymatic

365 activation function of Est1, the telomere recruitment or other function unique to Est1 may play a

366 role in TLC1-TLC1 association.

367

$368 \mathrm{Ku}$ and Sir4, but not Telomere Silencing or Tethering to the Nuclear Periphery, Promote the

369 Same Mode of TLC1-TLC1 Association 
371 To test whether other factors involved in telomerase recruitment to telomeres also affect TLC1-

372 TLC1 association, we first performed the coIP assays in Ku mutant strains. In contrast to the 373 more modest effects of the absence of essential telomerase components Est1, Est2 or Est3, 60 -

$37475 \%$ of the TLC1-TLC1 association was consistently lost in $y k u 70 \Delta$ and $y k u 80 \Delta$ strains, as well

375 as in $y k u 80-135 i$ strains (Figure 5D), which have a small insertion in Ku that specifically

376 abrogates TLC1-Ku interaction, but leaves NHEJ intact (Stellwagen et al., 2003). As previously 377 reported (Mozdy, Podell \& Cech, 2008), in all these Ku mutant strains the steady-state level of 378 total TLC1 was reduced by about $25-50 \%$ (Figure $5 \mathrm{E}$ ), and telomeres, while stable, are shorter 379 than in wild-type. Therefore we tested two different mutations (cdc73 4 , ctr9 9 ) that reduce the 380 steady-state level of TLC1 much more than the Ku mutations (Figure 5E). Neither $c d c 73 \Delta$ nor $381 \operatorname{ctr} 9 \Delta$ caused any decrease in the fraction of dimeric TLC1 (Figure 5D). Furthermore, two 382 mutations known to cause short telomeres (arfl $\Delta$ and tel1 $\Delta$ ) (Askree et al., 2004), also did not 383 reduce TLC1-TLC1 association (Figure 5D and E). The combined findings above indicate that $384 \mathrm{Ku}$ binding to TLC1 promotes or stabilizes TLC1-TLC1 association, and that neither reduction 385 in TLC1 steady state level nor shorter, stable telomeres is sufficient to impair TLC1-TLC1 386 association.

388 The $\mathrm{Ku}$ complex is also necessary for telomere silencing (Boulton \& Jackson, 1998) and 389 telomere tethering to the nuclear periphery (Taddei et al., 2004). However, by using mutations 390 that affect these processes, we found evidence that it is not because of these functions that $\mathrm{Ku}$

391 plays a role in TLC1-TLC1 association. Specifically, sir3 $\Delta$ (which abrogates telomere silencing) 392 and neither ctf184 nor esc1 $\Delta$ (which each diminish telomere tethering) (Hiraga, Robertson \& 393 Donaldson, 2006) decreased TLC1-TLC1 association levels (Figure 6A). In marked contrast, $394 \operatorname{sir} 4 \Delta$ as well as sir4-42 mutations diminished TLC1-TLC1 association to the same extent as $395 y k u 80-135 i$ and sir24 to a lesser extent (Figure 6A). SIR4-42 mutation truncates the C-terminus 396 of Sir4 and fails to recruit silent chromatin factors to telomeres (Kennedy et al., 1995). Sir4 is 397 distinguished from the other telomere silencing Sir proteins Sir2 and Sir3 by its localization on 398 telomeres closer to the distal tip than Sir2 and Sir3, and the Ku complex is reported to interact 399 physically with Sir4 (Tsukamoto, Kato \& Ikeda, 1997). Since Ku and Sir4 are localized on 400 telomeres, we tested whether detection of TLC1-TLC1 association in cell extracts by the coIP 
401 assay was dependent on DNA. However, DNase treatment of the extracts did not diminish the

402 fraction of TLC1 detected in dimeric form (Figure 6B).

403

404 To test if the Ku complex and Sir4 act in the same pathway for TLC1-TLC1 association, we

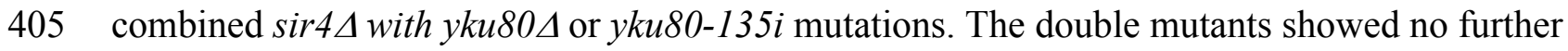
406 reduction in the TLC1 dimer fraction compared to single mutants (Figure 6C). We conclude that $407 \mathrm{Ku}$ binding to TLC1 and Sir4 regulates TLC1-TLC1 association through the same pathway, 408 which is independent of telomere silencing or anchoring to the nuclear periphery.

409

$410 \mathrm{Ku} / \mathrm{Sir} 4$ and the 3'-Cleaved TLC1 Precursor Sequence Promote TLC1-TLC1 association by 411 Different Modes

412

413 To determine the relationship between the roles of $\mathrm{Ku} / \mathrm{Sir} 4$ and the 3' region of TLC1 in TLC1414 TLC1 association, we combined sir4 $\Delta$ or $y k u 80 \Delta$ mutation with the 3' mutation $t l c 1-42 G$. In 415 these double mutants (sir4 $\Delta t l c 1-42 G$ and $y k u 80 \Delta$ tlcl-42G strains), compared to either each 416 single-mutant strain or the sir4 $4 y k u 80 \Delta$ double mutant, the TLC1-TLC1 association was further 417 reduced, down to almost to the background control level (Figure 6D). This indicated that the 418 TLC1-TLC1 association that is dependent on the 3' region of TLC1 is at least partially 419 independent of $\mathrm{Ku}$ and Sir4, possibly mediated by a different pathway.

420

Lack of Evidence for Est2-Est2 Physical Association

422

423 Although, as described above, we did not find evidence that TLC1-TLC1 association was highly 424 dependent on Est2, we tested the possibility that any of the small fraction of TLC1-TLC1 425 association that may be potentially affected by Est 2 deletion might be mediated through association of one Est 2 molecule with another Est 2 molecule. To this end, we performed four different assays in attempts to detect any such physical Est2-Est2 interaction in vivo. First, we attempted to detect Est2-Est2 interaction by yeast two-hybrid assay in which Est2 was fused to the Gal4 activation domain and DNA binding domain separately; such assays showed no positive

430 signals for Est2-Est2 interaction. Secondly, we co-expressed Est2-FLAG and Est2-myc and 431 performed co-immunoprecipitation assays; however, no signal indicative of co- 
432 immunoprecipitation was detected in the Western blots in these experiments. Thirdly, to 433 overcome the potential issues of the detection limit using Western blotting, we performed coIP 434 experiments using presence of TLC1 as a proxy signal, via qRT-PCR assays as described above. 435 In this approach, we co-expressed wild-type Est2-HA with either wild-type Est2-myc (positive 436 control) or est2 $\triangle C P$-myc. est2 $\triangle C P$ deletes the Est2 domain that is required for Est2-TLC1 437 interaction (Lin \& Blackburn, 2004). Therefore, the presence of an interaction between Est2-HA 438 and Est2 $\triangle C P$-myc can be ascertained by proxy using the measurement of TLC1 in est $2 \triangle C P$-myc 439 IP. However, we did not observe any such enrichment of TLC1 in this coIP assay (Figure 7A). 440 Finally, because TLC1 detection by the qRT-PCR assay had high sensitivity, we also performed 441 sequential coIP experiments with strains co-expressing Est2-FLAG and Est2-myc. In this assay, 442 Est2-FLAG was adsorbed onto anti-FLAG gel matrix and subsequently eluted with FLAG 443 peptide, and any Est2-myc present in the elution fraction was immunoprecipitated with anti-myc 444 antibody. The amount of TLC1 was then quantified in this final immunoprecipitate; while the 445 positive control (Est2-FLAG-myc) showed robust enrichment, we found no enrichment of TLC1 446 compared to the negative control (Figure 7B). We conclude that, although the possibility of a 447 weak or transient association between Est2 molecules cannot be ruled out, these negative lines of 448 evidence are consistent with the model that the majority of the TLC1-TLC1 in vivo association is independent of an active telomerase enzyme complex.

450

451

452

453 454 455 456 457 458 459 460 461

\section{DISCUSSION}

Here we have explored the nature of telomerase RNA-RNA associations in vivo in S. cerevisiae. We report that $\sim 10 \%$ of the TLC1 molecules in vivo are physically associated with another TLC1 molecule. We refer to this as TLC1-TLC1 association for simplicity, although the data do not formally exclude the possibility of higher oligomerization forms. The lack of formation of TLC1-TLC1 association in lysates (the mix experiments) suggest that either TLC1 level is too low in the lysate or there is an active mechanism required for the association. This TLC1-TLC1 association increases by two-fold specifically in G1 phase of the cell cycle, and takes place via two distinguishable modes. 
462 First, mutating a sequence in the 3' region of TLC1 that is cleaved off during the production of 463 the mature form of TLC1 reduced TLC1-TLC1 association by about half. The TLC1-TLC1 464 association of both the mature and the immature TLC1 forms were comparably affected by this 465 3' sequence mutation. This same sequence has previously been implicated in TLC1-TLC1 466 association in vitro and its mutation shown to shorten telomeres (Gipson et al., 2007). Our 467 findings thus indicate this 3' sequence-dependent mode of TLC1-TLC1 association occurs in 468 vivo during telomerase biogenesis. This is further consistent with our findings that TLC1-TLC1 469 association depends on nuclear export to the cytoplasm, where biogenesis of telomerase is 470 reported to occur, and that TLC1-TLC1 association increases in G1 phase, the only time in the 471 cell cycle when TLC1 maturation cleavage is active (Chapon, Cech \& Zaug, 1997).

472

473 The second mode of TLC1-TLC1 association requires $\mathrm{Ku}$ binding to TLC1; mutations 474 preventing $\mathrm{Ku}$-TLC1 interaction reduced TLC1-TLC1 association by about half. The Ku475 associated protein Sir4 was also required for this mode. The Sir and Ku complexes are both important factors in maintaining telomeres; their functions include forming silent chromatin at telomeres and recruiting telomeres to nuclear periphery (Boulton \& Jackson, 1998; Taddei et al., 478 2004). Interestingly however, although Sir4 is part of the silent information regulator Sir complex, TLC1-TLC1 association required neither classic silencing (neither Sir2 nor Sir3 was required), nor Ku-mediated telomere tethering to the nuclear periphery (neither Esc1 nor Ctf18 was required).

482

483 The additive genetic disruptions of these two modes of in vivo TLC1-TLC1 association - RNA sequence mutations in the 3' region of TLC1 and deletion of the protein factors Ku and Sir4 have an intriguing parallel to the in vitro disruptions of TLC1-TLC1 association in the immunoprecipitate, via either competition with excess oligonucleotides (most sensitive in the 3' region) or protease treatment. Each of these two in vitro treatments disrupted only a fraction of the TLC1-TLC1 association. Combining these findings, the simplest interpretation is that these two fractions correspond to or overlap with the TLC1 3' sequence-dependent and the Ku/Sir4 dependent association modes respectively. 
492 Simultaneously mutating both the 3' precursor TLC1 sequence and abrogating Ku-TLC1 binding

493 abolished in vivo TLC1-TLC1 association to background levels. The epistasis analyses together

494 indicate that for physical TLC1-TLC1 association, Ku and Sir4 act in the same pathway, which

495 is distinct from the pathway requiring the 3' end sequence of the immature TLC1 RNA. Notably,

496 each of the various kinds of mutations that we report here to impair TLC1-TLC1 association also

497 causes telomeres to be shorter than wild-type (Askree et al., 2004), consistent with TLC1-TLC1

498 association in vivo having functional significance.

499

500 Our findings indicate two separable and potentially independent modes of TLC1-TLC1

501 association - the first involving the TLC1 3' region prior to cleavage to the mature form, and a

502 subsequent mode involving Ku/Sir4. We propose a model (Figure 8) by which all TLC1

503 molecules transiently engage in TLC1-TLC1 association during at least two stages in telomerase

504 biogenesis. The first TLC1-TLC1 association mode occurs prior to TLC1 maturation and

505 requires a sequence in the 3'extension of the TLC1 precursor (Figure 8 Mode 1). The lack of

506 compensatory mutation recue of TLC1-TLC1 association suggests the palindromic sequence in

507 the 3' region may be important for a recruitment of a factor or a secondary structure that is

508 important in TLC1-TLC1 association rather than trans base-pairing. The TLC1-TLC1

509 association is further stabilized by RNA-RNA or RNA-protein interactions that persist after

510 TLC1 cleavage/maturation, which can be partially disrupted in vitro by anti-sense

511 oligonucleotides - particularly those complementary to the 3' region of the mature telomerase

512 RNA. Our findings suggest that multiple regions of TLC1 RNA help stabilize the TLC1-TLC1

513 association, and are consistent with a model of their "unzipping" caused by the addition of

514 competing oligonucleotides.

515

516 The second mode requires Ku complex binding to TLC1 and also depends on Sir4 (Figure 8

517 Mode II). While it is not known when in the biogenesis and maturation of TLC1 Ku (and

518 possibly $\mathrm{Ku}$-bound Sir4) become associated with TLC1, Ku and Sir4 are both thought to

519 function at telomeres, where the vast majority of TLC1 (>95\%) is already processed to the

520 mature form (i.e. missing the 3' region). Both mature TLC1 and uncleaved precursor TLC1 were

521 found coIP'ed with Est2, albeit with the IP efficiency of the immature form being reduced by

522 about half (Figure 2G). Thus, cleaving off the 3' region of TLC1 is not an obligatory step for 
523 TLC1 in order for it to engage in telomerase enzyme complex formation. This is consistent with

524 the lack of interdependence we found between the 3'sequence-mediated association during

$525 \mathrm{TLC} 1$ biogenesis and the $\mathrm{Ku} / \mathrm{Sir}$-dependent association.

526

527 Interestingly, some of the data presented here cannot easily be reconciled with the data

528 previously reported. Specifically, Gipson et al reported that the compensatory mutations in the 3'

529 palindromic sequence ( $t l c 1-42 G$ and $t l c 1-42 C$ ) dimerized in vitro and rescued telomere

530 shortening in trans; however, we observed that the in vivo TLC1-TLC1 association is not

531 rescued by trans compensatory mutations. We observed no in vitro TLC1-TLC1 association in

532 lysates (Figure 2E), while Gipson et al showed that high concentrations of in vitro transcribed

533 TLC1 readily dimerized in vitro. These contrasting observations suggest that in vitro and in vivo

534 TLC1-TLC1 associations may results from different mechanisms. The rescue of telomere length

535 in strains co-expressing both $t l c 1-42 G$ and $t l c 1-42 C$ observed by Gipson et al may have resulted

536 from increased total TLC1 levels compared to strains expressing $t l c 1-42 G$ or $t l c 1-42 C$ alone.

537 Hence, these observations are not directly contradictory; however further studies delineating the

538 importance of in vivo TLC1-TLC1 association with telomerase functions should clarify these

539 seemingly contradictory findings.

540

541 Lin and Blackburn reported physical interactions between Est2 molecules by differentially

542 tagging two copies of Est 2 in coIP assays. The same strains were used in this study to test for

543 presence of Est2-Est2 interaction by measuring TLC1 levels as a proxy. Surprisingly, in contrast

544 to published results, TLC1 did not coimmunoprecipitate. It is possible that TLC1 only interacts

545 with monomeric Est2, and that dimeric Est2's are inactive.

547 Finally, the presence of two independent modes and machineries for TLC1-TLC1 association

548 suggest that such interaction reflects an important aspect of yeast telomere maintenance biology;

549 a conclusion reinforced by the telomere shortening that results from all the mutations that

550 disrupted TLC1-TLC1 association. However, this report leaves open the detailed mechanisms of

551 these novel in vivo TLC1-TLC1 physical association modes that we have demonstrated in this

552 study. One speculation is that these RNA-RNA associations may be important for the stability of

553 telomerase RNA as it is shuttled among cytoplasmic and nuclear compartments for various 
554 maturation steps; a possible model is that TLC1-TLC1 association assists the RNA in acting as

555 its own chaperone. We can further speculate that this might be an important regulatory step for

556 telomerase activity, as the yeast telomerase holoenzyme shows no physical evidence of

557 oligomerization. For example, a dissociation of TLC1-TLC1 association, which likely requires

558 energy, may act as a switch mechanism for forming a fully competent telomerase holoenzyme.

559 There are also other cell cycle regulated telomerase activation factors such as Estl and Ku that

560 recruit telomerase complex to the telomere at different stages of cell cycle, and it is of great

561 interest to test how these factors may affect TLC1-TLC1 associations in cell cycle-dependent

562 manner. Further research will be needed to decipher the mechanistic and functional significance

563 of intermolecular interactions among telomerase components.

\section{ACKNOWLEDGEMENTS}

567 The authors thank Tracy Chow, Beth Cimini, Kyle Jay, Jue Lin, Imke Listerman, and Dana

568 Smith for critical reading of the manuscript and helpful discussion.

569

570

\section{REFERENCES}

571 Alves D., Li H., Codrington R., Orte A., Ren X., Klenerman D., Balasubramanian S. 2008. Single-molecule analysis of human telomerase monomer. Nat Chem Biol 4:287-289.

573

574

575

576

577

578

579

580
Askree SH., Yehuda T., Smolikov S., Gurevich R., Hawk J., Coker C., Krauskopf A., Kupiec M., McEachern MJ. 2004. A genome-wide screen for Saccharomyces cerevisiae deletion mutants that affect telomere length. Proceedings of the National Academy of Sciences of the United States of America 101:8658-8663.

Baker Brachmann C., Davies A., Cost GJ., Caputo E., Li J., Hieter P., Boeke JD. 1998. Designer deletion strains derived from Saccharomyces cerevisiae S288C: A useful set of strains and plasmids for PCR - mediated gene disruption and other applications. Yeast 14:115132. 
581 Beattie TL., Zhou W., Robinson MO., Harrington L. 2001. Functional Multimerization of the

582 Human Telomerase Reverse Transcriptase. Mol. Cell. Biol. 21:6151-6160.

583 Boulton SJ., Jackson SP. 1998. Components of the Ku-dependent non-homologous end-joining 584 pathway are involved in telomeric length maintenance and telomeric silencing. The 585 EMBO Journal 17:1819-1828. Molecular Biology of the Cell 14:4794-4804.

Chan A., Boulé J-B., Zakian VA. 2008. Two Pathways Recruit Telomerase to Saccharomyces cerevisiae Telomeres. PLoS Genet 4:e1000236.

Chapon C., Cech TR., Zaug AJ. 1997. Polyadenylation of telomerase RNA in budding yeast. RNA (New York, N.Y.) 3:1337-1351.

Evans SK., Lundblad V. 2002. The Est1 Subunit of Saccharomyces cerevisiae Telomerase Makes Multiple Contributions to Telomere Length Maintenance. Genetics 162:11011115.

Fisher TS., Taggart AKP., Zakian VA. 2004. Cell cycle-dependent regulation of yeast telomerase by Ku. Nat Struct Mol Biol 11:1198-1205.

597 Franke J., Gehlen J., Ehrenhofer-Murray AE. 2008. Hypermethylation of yeast telomerase RNA by the snRNA and snoRNA methyltransferase Tgs1. Journal of Cell Science 121:35533560.

600 Gallardo F., Olivier C., Dandjinou AT., Wellinger RJ., Chartrand P. 2008. TLC1 RNA nucleo-

601 cytoplasmic trafficking links telomerase biogenesis to its recruitment to telomeres.

602 EMBO J 27:748-757. 
603 Gipson CL., Xin Z-T., Danzy SC., Parslow TG., Ly H. 2007. Functional Characterization of 604 Yeast Telomerase RNA Dimerization. Journal of Biological Chemistry 282:18857 $605 \quad 18863$.

606 Hiraga S., Robertson ED., Donaldson AD. 2006. The Ctf18 RFC-like complex positions yeast 607 telomeres but does not specify their replication time. EMBO J 25:1505-1514.

608 Jain D., Cooper JP. 2010. Telomeric strategies: means to an end. Annual Review of Genetics 609 44:243-269.

610 Jiang J., Miracco EJ., Hong K., Eckert B., Chan H., Cash DD., Min B., Zhou ZH., Collins K., 611 Feigon J. 2013. The architecture of Tetrahymena telomerase holoenzyme. Nature $612 \quad 496: 187-192$.

613 Kennedy BK., Austriaco Jr. NR., Zhang J., Guarente L. 1995. Mutation in the silencing gene 614 S/R4 can delay aging in S. cerevisiae. Cell 80:485-496.

615 de Lange T., Lundblad V., Blackburn E. 2006. Telomeres, Second Edition. Cold Spring Harbor $616 \quad$ Laboratory Press.

617 Lin J., Ly H., Hussain A., Abraham M., Pearl S., Tzfati Y., Parslow TG., Blackburn EH. 2004. A 618 universal telomerase RNA core structure includes structured motifs required for binding 619 the telomerase reverse transcriptase protein. Proceedings of the National Academy of $620 \quad$ Sciences of the United States of America 101:14713-14718.

621 Lin J., Blackburn EH. 2004. Nucleolar protein PinX1p regulates telomerase by sequestering its 622 protein catalytic subunit in an inactive complex lacking telomerase RNA. Genes \& Development 18:387-396.

624 Mozdy AD., Cech TR. 2006. Low abundance of telomerase in yeast: Implications for telomerase 625 haploinsufficiency. RNA 12:1721 -1737. 
626 Mozdy AD., Podell ER., Cech TR. 2008. Multiple Yeast Genes, Including Pafl Complex Genes,

627

628

629

630

631

632

633

634

635

636

637

638

639

640

641

642

643

644

645

646

647

648

Affect Telomere Length via Telomerase RNA Abundance. Mol. Cell. Biol. 28:41524161.

Osterhage JL., Talley JM., Friedman KL. 2006. Proteasome-dependent degradation of Est1p regulates the cell cycle-restricted assembly of telomerase in Saccharomyces cerevisiae. Nat Struct Mol Biol 13:720-728.

Prescott J., Blackburn EH. 1997. Functionally interacting telomerase RNAs in the yeast telomerase complex. Genes \& Development 11:2790 -2800.

Sauerwald A., Sandin S., Cristofari G., Scheres SHW., Lingner J., Rhodes D. 2013. Structure of active dimeric human telomerase. Nature structural \& molecular biology 20:454-460.

Seto AG., Livengood AJ., Tzfati Y., Blackburn EH., Cech TR. 2002. A bulged stem tethers Est1p to telomerase RNA in budding yeast. Genes \& Development 16:2800 -2812.

Shcherbakova DM., Sokolov KA., Zvereva MI., Dontsova OA. 2009. Telomerase from yeast Saccharomyces cerevisiae is active in vitro as a monomer. Biochemistry (Moscow) $74: 749-755$.

Stellwagen AE., Haimberger ZW., Veatch JR., Gottschling DE. 2003. Ku interacts with telomerase RNA to promote telomere addition at native and broken chromosome ends. Genes \& Development 17:2384-2395.

Taddei A., Hediger F., Neumann FR., Bauer C., Gasser SM. 2004. Separation of silencing from perinuclear anchoring functions in yeast Ku80, Sir4 and Esc1 proteins. EMBO J 23:1301-1312.

Teixeira MT., Forstemann K., Gasser SM., Lingner J. 2002. Intracellular trafficking of yeast telomerase components. EMBO Reports 3:652-659. 
649 Tomlin GC., Wixon JL., Bolotin-Fukuhara M., Oliver SG. 2001. A new family of yeast vectors 650 and S288C-derived strains for the systematic analysis of gene function. Yeast 651 (Chichester, England) 18:563-575.

652 Tsukamoto Y., Kato J., Ikeda H. 1997. Silencing factors participate in DNA repair and 653 recombination in Saccharomyces cerevisiae. Nature 388:900-903.

654 Wang L., Dean SR., Shippen DE. 2002. Oligomerization of the telomerase reverse transcriptase 655 from Euplotes crassus. Nucleic Acids Research 30:4032-4039.

656 Wenz C., Enenkel B., Amacker M., Kelleher C., Damm K., Lingner J. 2001. Human telomerase 657 contains two cooperating telomerase RNA molecules. The EMBO Journal 20:3526-3534.

658 Zappulla DC., Cech TR. 2004. Yeast telomerase RNA: A flexible scaffold for protein subunits. 659 Proceedings of the National Academy of Sciences of the United States of America $660 \quad 101: 10024-10029$.

661 


\section{Table $\mathbf{1}$ (on next page)}

Strains used

All strains are in the S288c strain background and are isogenic to BY4746 (Tomlin et al., 2001), except as noted below. BAR1 gene was deleted from BY4720 strain and mated to BY4741 (Baker Brachmann et al., 1998). The resulting diploid strain became the parental strain for two independent spores, yEHB22,321/465 and yEHB22,322/466. The strains yEHB22,803/804 were generous gift of Jonathan Weissman. All haploid strains have independently isolated isogenic duplicates from parental strains as noted in the table below (denoted by $y E H B 22, X X X / Y Y Y$, which refers to two stains yEHB22,XXX and yEHB22,YYY). The diploid strains, yEHB22,825-827 were created by mating yEHB321/465 and yEHB22,322/466 strains that have Est2 fused to indicated tags. Strains that were further modified in this study are in bold. 
1 Table 1. Strains used.

2

3 All strains are in the S288c strain background and are isogenic to BY4746 (Tomlin et al., 2001),

4 except as noted below. BAR1 gene was deleted from BY4720 strain and mated to BY4741

5 (Baker Brachmann et al., 1998). The resulting diploid strain became the parental strain for two

6 independent spores, yEHB22,321/465 and yEHB22,322/466. The strains yEHB22,803/804 were

7 generous gift of Jonathan Weissman. All haploid strains have independently isolated isogenic

8 duplicates from parental strains as noted in the table below (denoted by $y E H B 22, X X X / Y Y Y$,

9 which refers to two stains yEHB22,XXX and yEHB22,YYY). The diploid strains, yEHB22,825-

10827 were created by mating yEHB321/465 and yEHB22,322/466 strains that have Est 2 fused to

11 indicated tags. Strains that were further modified in this study are in bold.

\begin{tabular}{|c|c|}
\hline Strain number & Relevant genotype \\
\hline уЕНB22,321/465 & 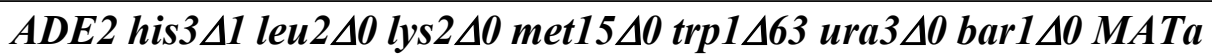 \\
\hline уЕНB22,322/466 & ADE2 his3 $\Delta 1$ leu $2 \Delta 0$ lys $2 \Delta 0$ met15 $\Delta 0$ trp1 $\Delta 63$ ura3 $\Delta 0$ bar1 $\Delta 0$ MATa \\
\hline yEHB22,495/496 & yEHB22,321/465 but $T L C 1-M S 2$ \\
\hline yEHB22,720/721 & yEHB22,321/465 but $H I S 3-P_{C Y C 1}-C P-3 x M y c$ \\
\hline yEHB22,722/723 & yEHB22,720/721 but TLC1-MS2 \\
\hline yEHB22,662/663 & yEHB22,720/721 but $T L C 1-U R A 3-T L C 1-M S 2$ \\
\hline yEHB22,750/751 & yEHB22,720/721 but $T L C 1-L E U 2-T L C 1-M S 2$ \\
\hline yEHB22,742/743 & yEHB22,720/721 but tlc1-42G-URA3-TLC1-MS2 \\
\hline yEHB22,744/745 & yEHB22,720/721 but tlc1-42C-URA3-TLC1-MS2 \\
\hline yEHB22,776/777 & yEHB22,720/721 but $t l c 1-42 C-U R A 3-t l c 1-42 G-M S 2$ \\
\hline yEHB22,704/705 & yEHB22,662/663 but $\operatorname{tgs} 1 \triangle::$ KanMX6 \\
\hline yEHB22,768/769 & yEHB22,750/751 but nup 133А::KanMX6 \\
\hline yEHB22,698/699 & yEHB22,662/663 but est $1 \triangle:: K a n M X 6$ \\
\hline yEHB22,724/725 & yEHB22,662/663 but est $2 \triangle:: K a n M X 6$ \\
\hline yEHB22,700/701 & yEHB22,662/663 but est $3 \triangle:: K a n M X 6$ \\
\hline yEHB22,682/683 & yEHB22,662/663 but $y k u 70 \Delta:: K a n M X 6$ \\
\hline yEHB22,686/687 & yEHB22,662/663 but $y k u 80 \Delta:: K a n M X 6$ \\
\hline yEHB22,758/759 & yEHB22,750/751 but $y k u 80-135 i$ \\
\hline yEHB22,702/703 & yEHB22,662/663 but arf1 $\triangle:: K a n M X 6$ \\
\hline yEHB22,706/707 & yEHB22,662/663 but $c d c 73 \Delta::$ KanMX6 \\
\hline yEHB22,726/727 & yEHB22,662/663 but ctr9A::KanMX6 \\
\hline yEHB22,764/765 & yEHB22,750/751 but ctf184::KanMX6 \\
\hline yEHB22,766/767 & yEHB22,750/751 but esc $1 \triangle::$ KanMX6 \\
\hline yEHB22,728/729 & yEHB22,662/663 but $\operatorname{sir} 2 \Delta:: K a n M X 6$ \\
\hline yEHB22,762/763 & yEHB22,750/751 but sir3 $\triangle::$ KanMX6 \\
\hline yEHB22,730/731 & yEHB22,662/663 but $\operatorname{sir} 4 \Delta::$ KanMX6 \\
\hline yEHB22,787/788 & yEHB22,662/663 but sir4-42::KanMX6 \\
\hline yEHB22,770/771 & yEHB22,750/751 but tell $\Delta:: K a n M X 6$ \\
\hline yEHB22,774/775 & yEHB22,662/663 but sir4A::KanMX6 yku80A::TRP1 \\
\hline yEHB22,776/777 & yEHB22,720/721 but tlc1-42G-URA3-TLC1-MS2 yku80A::TRP1 \\
\hline yEHB22,803/804 & LYS2 can1 $1:: S T E 2{ }_{P}-H I S 5$ lyp1 $1:: S T E 3_{P}-L E U 2 M A T \alpha$ \\
\hline
\end{tabular}




\begin{tabular}{|c|c|}
\hline yEHB22,805/806 & yEHB22,803/804 but TLC1-MS2 \\
\hline yEHB22,807/808 & yEHB22,803/804 but $T L C 1-U R A 3-T L C 1-M S 2$ \\
\hline yEHB22,809/810 & yEHB22,803/804 but tlc1-42G-URA3-TLC1-MS2 \\
\hline yEHB22,811/812 & yEHB22,803/804 but tlc1-42C-URA3-TLC1-MS2 \\
\hline yEHB22,813/814 & yEHB22,803/804 but $t l c 1-42 C-U R A 3-t l c 1-42 G-M S 2$ \\
\hline yEHB22,815/816 & yEHB22,807/808 but $y k u 80-135 i$ \\
\hline yEHB22,817/818 & yEHB22,807/808 but sir4 $\triangle::$ KanMX6 \\
\hline yEHB22,819/820 & yEHB22,807/808 but $\operatorname{sir} 2 \triangle::$ KanMX6 \\
\hline yEHB22,821/822 & yEHB22,807/808 but sir4 $4::$ KanMX6 yku80-135i \\
\hline yEHB22,823/824 & yEHB22,803/804 but tlc1-42G-URA3-TLC1-MS2 sir4A::KanMX6 \\
\hline yEHB22,825 & EST2-3xFLAG/EST2-13xMyc MATa/a \\
\hline yEHB22,826 & EST2-3xFLAG/EST2 MATa/ \\
\hline yEHB22,827 & EST2-3xFLAG-13xMyc/EST2 MATa/ $\alpha$ \\
\hline
\end{tabular}


Table 2 (on next page)

Primer sequences for qRT-PCR 
1 Table 2. Primer sequences for qRT-PCR

2

\begin{tabular}{|c|c|c|}
\hline Amplicon & Primer number & Sequence $\left(5^{\prime}\right.$ to 3 ') \\
\hline \multirow[t]{2}{*}{$P G K 1$} & oEHB22,0716 & GGCTGGTGCTGAAATCGTTCCAAA \\
\hline & oEHB22,0717* & AGCCAGCTGGAATACCTTCCTTGT \\
\hline \multirow[t]{2}{*}{ Untagged $T L C 1$} & oEHB22,0561 & CATCGAACGATGTGACAGAGAA \\
\hline & oEHB22,0801* & GACAAAAATACCGTATTGATCATTAA \\
\hline \multirow{2}{*}{$\begin{array}{l}\text { MS2-tagged } \\
\text { TLC1 }\end{array}$} & oEHB22,0563 & ATGCCTGCAGGTCGACTCTAGAAA \\
\hline & oEHB22,0338* & TGCGACAAAAATACCGTATTGATCA \\
\hline \multirow{2}{*}{$\begin{array}{l}\text { Uncleaved, } \\
\text { untagged } T L C 1\end{array}$} & oEHB22,1015 & TATCTATTAAAACTACTTTGATGATCAGTA \\
\hline & oEHB22,1038* & AGCGATATACAAGTACAGTACGCGCG \\
\hline Uncleaved, & oEHB22,0339 & AGCTTGCATGCCTGCAGGTCGACTC \\
\hline $\begin{array}{l}\text { MS2-tagged } \\
\text { TLC1 }\end{array}$ & oEHB22,1038* & AGCGATATACAAGTACAGTACGCGCG \\
\hline \multirow[t]{2}{*}{ CLN2 } & oEHB22,712 & TTGTTCGAGCTGTCTGTGGTCACT \\
\hline & oEHB22,713* & AATTTGGCTTGGTCCCGTAACACG \\
\hline \multirow[t]{2}{*}{ CLN3 } & oEHB22,837 & AAGGCCGCTGTACAACCTGACTAA \\
\hline & oEHB22,838* & TGAACCGCGAGGAATACTTGTCCA \\
\hline
\end{tabular}

3

4 *Primer used in the reverse transcription step 


\section{Figure $\mathbf{1}$ (on next page)}

TLC1-TLC1 features and MS2 hairpin insertion

1A. Schematic and linear maps of relevant features of TLC1 RNA coding sequence before it is polyadenylated and cleaved (+1-1301). TmG: 5' trimethylG cap ( (Franke, Gehlen \& Ehrenhofer-Murray, 2008) ); The binding sites for Ku (+288-335), Est1 (+660-664), and Sm $(+1153-1160)$ proteins are indicated. Telomeric template: $(+468-484) ;$ CS3 $(+719-784)$ and CS4 (+785-853): two sequences, conserved in budding yeasts, that form two sides of a stem of the evolutionarily conserved telomerase RNA pseuduoknot structure ( (Lin et al., 2004) ); MS2: site of the tandem inserted MS2 coat protein-binding hairpins used in this work, at Bcll site (+1033); cleavage site: the 3' end of the mature TLC1 (+1167); CGCGCG: sequence $(+1204)$ previously implicated in TLC1 in vitro dimerization, located in the cleaved-off 3' extension of pre-processed immature TLC1 RNA ( (Chapon, Cech \& Zaug, 1997) ). 1B. Antisense oligonucleotides targeted against the full length of TLC1. Each of the 72 anti-sense oligonucleotides are 30 bases in length and overlap with each other by 2-5 bases. The oligos are divided into 9 groups (alternating set of blue and red) of 8 oligos. 1C. The MS2 RNA binding hairpins were inserted at $\mathrm{Ncol}$ (nucleotide position 455) or Bcll (nucleotide position 1033) of TLC1 RNA coding gene. The telomere Southern blot of $Y^{\prime}$ telomeres shows the impact of the MS2 insert at different positions. The table at the top denotes where the MS2 was inserted (at either nucleotide positions 455 or 1033). $F$ and R denote whether the MS2 hairpin was inserted in correct (F) or in reverse (R) direction. The MS2 insertion at 1033 position seem to have the least impact on the telomere length (yEHB22,495/496). WT = yEHB22,321/465. 
A.

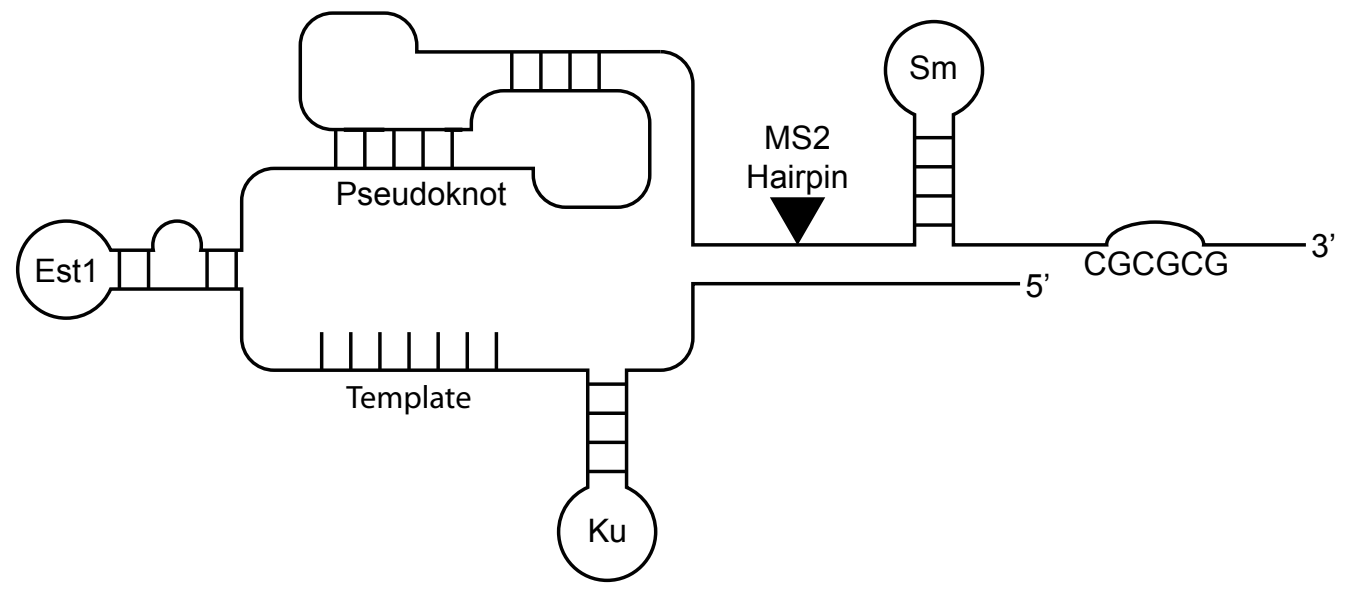

B.

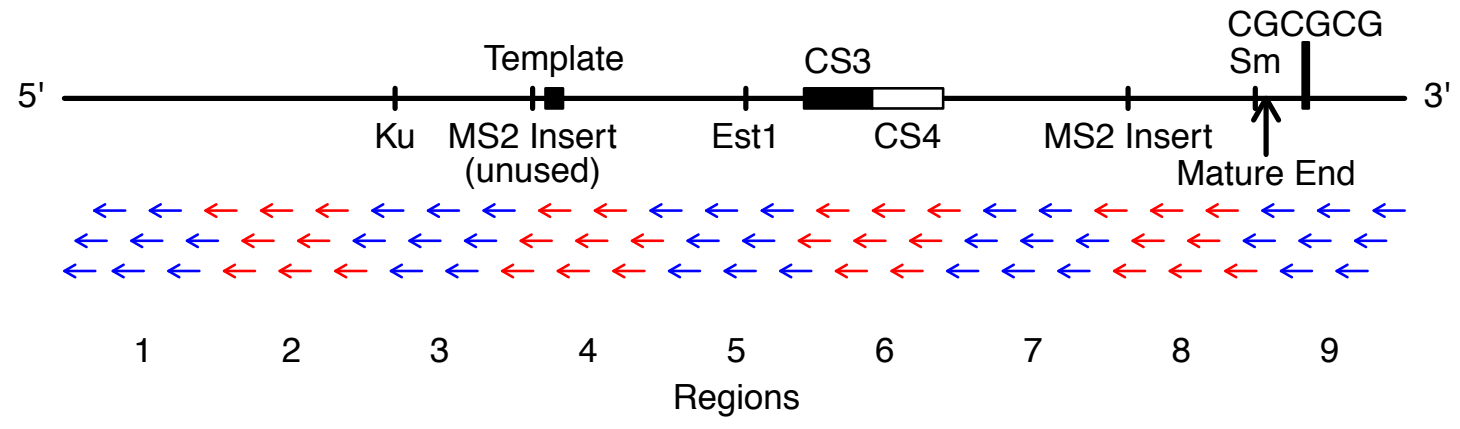

C.

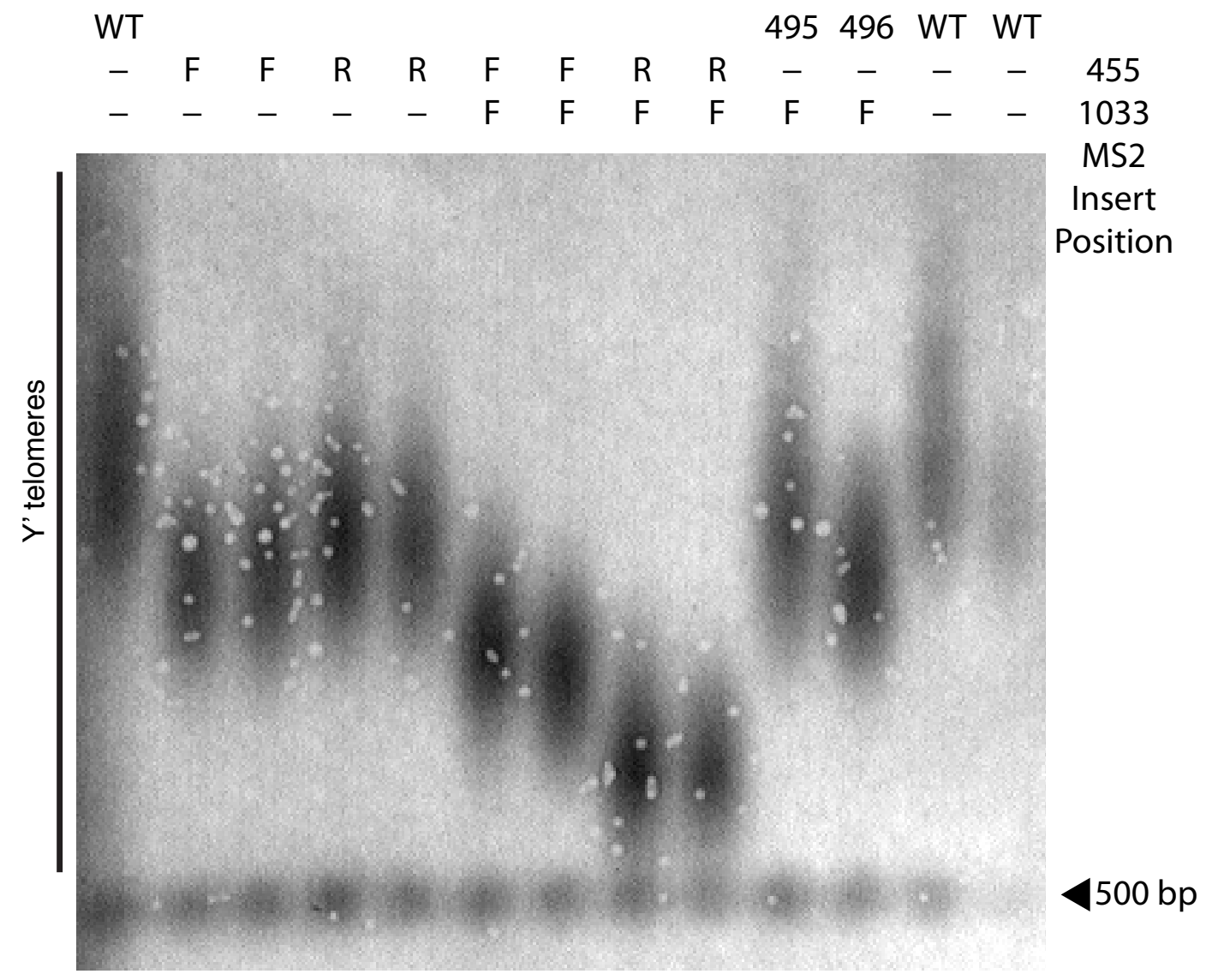




\section{Figure 2 (on next page)}

\section{Detection of TLC1-TLC1 Association}

2A. Primers used for specifically quantifying untagged or MS2-tagged TLC1 by qRT-PCR is shown schematically. The sequences of these primers are presented in Table 2 . The bulge represent the MS2 sequence inserted at the Bcll site of TLC1. The forward primer for specifically quantifying untagged TLC1 spans the insertion site of MS2. 2B. The amounts of untagged or MS2-tagged TLC1 were quantified in strains expressing only untagged TLC1 (yEHB22,321/465) or only MS2-tagged TLC1 (yEHB22,495/496). The lysates were also mixed $1: 1$ in the last two groups (yEHB22,321+yEHB22,495 and yEHB22,465+yEHB22,496). PGK1 values were normalized to the average of all samples. Untagged and MS2-tagged TLC1 values were normalized to the averages of yEHB22,321/465 and yEHB22,495/496, respectively. The error bars indicate the standard deviation of technical triplicates. The numbers below the $x$-axis represent the "yEHB22,xxx" strains, and the table below indicates the presence of untagged or MS2-tagged TLC1 in each sample. 2C. The amounts of untagged and MS2-tagged TLC1 in total RNA normalized to PGK1 mRNA level are shown. TLC1 was expressed from the genomic locus (yEHB22,662/663) or on a CEN-ARS plasmid (pRS416-TLC1 in yEHB22,722/723). MS2-tagged TLC1 were expressed from the genomic locus. In "Mix" samples, two strains expressing tagged (yEHB22,722/723) and untagged TLC1 (yEHB22,720/721) independently were mixed before the lysis step. The error bars indicate standard deviation from two experiments. 2D. The MS2 -tagged TLC1 associated with Est2. The fraction of tagged and untagged TLC1 coimmunoprecipitated with Myc tagged Est2 was quantified and normalized to the untagged TLC1 fraction. The lysates from yEHB22,321/465 strains carrying pRS426-EST2-Myc was used. The error bar indicates the standard deviation between two experiments. 2E. Detection of TLC1-TLC1 association by the coimmunoprecipitation strategy. The amount of untagged TLC1 co-immunoprecipitated with MS2-tagged TLC1 was used to estimate the fraction of total TLC1 that is dimeric. The

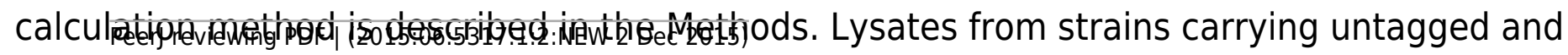


MS2-tagged TLC1 were used (WT = yEHB22,662/663). As negative controls, separate strains carrying either MS2-tagged or untagged TLC1 (MS2-tagged = yEHB22,722/723,

yEHB22,805/806, untagged $=$ yEHB22,720/721) were mixed just before lysis. The error bars indicate the standard errors among the experiments, and the numbers just below the $x$-axis denote the number of experiments performed. $2 \mathrm{~F}$. The immature TLC1 molecules accounts for only $4-8 \%$ of the total level of TLC1 molecules, and that this fraction was unchanged in the co-immunoprecipitated versus total TLC1. The lysates from the strains yEHB22,662/663 were used. The immature TLC1 quantity was divided by the total TLC1 amount, and the ratio was normalized to the input ratio. The error bars indicate standard deviation between two experiments. 2G. The immature TLC1 associated with Est2. The fraction of TLC1, total or immature, coimmunoprecipitated with FLAG-tagged Est2 was quantified and normalized to the total TLC1 fraction. The lysates from yEHB22,826. The error bar indicates the standard deviation between two experiments. 
A.

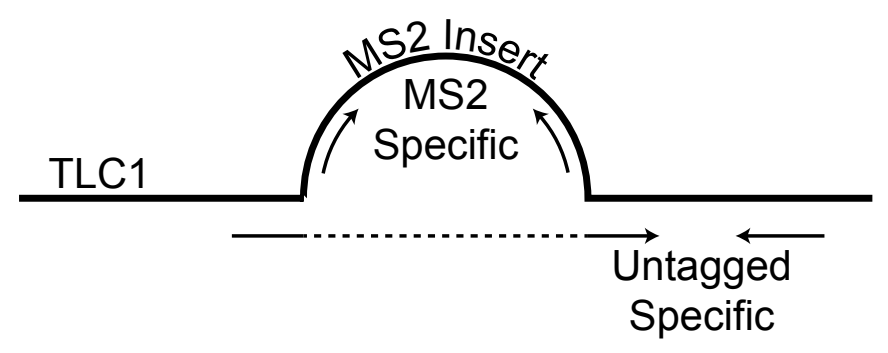

B.

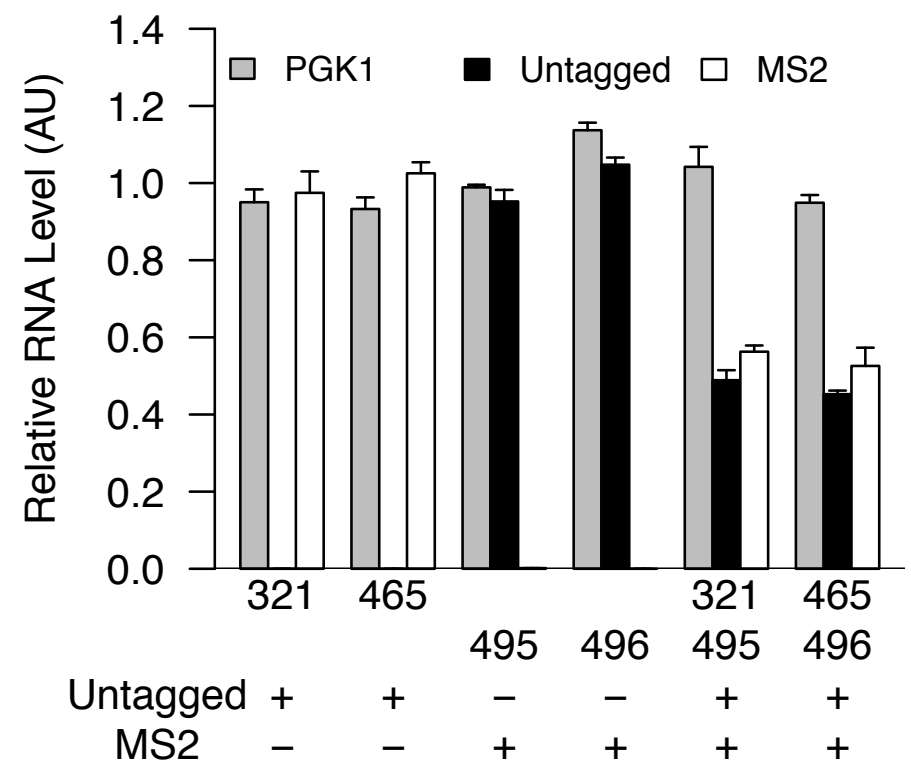

D.

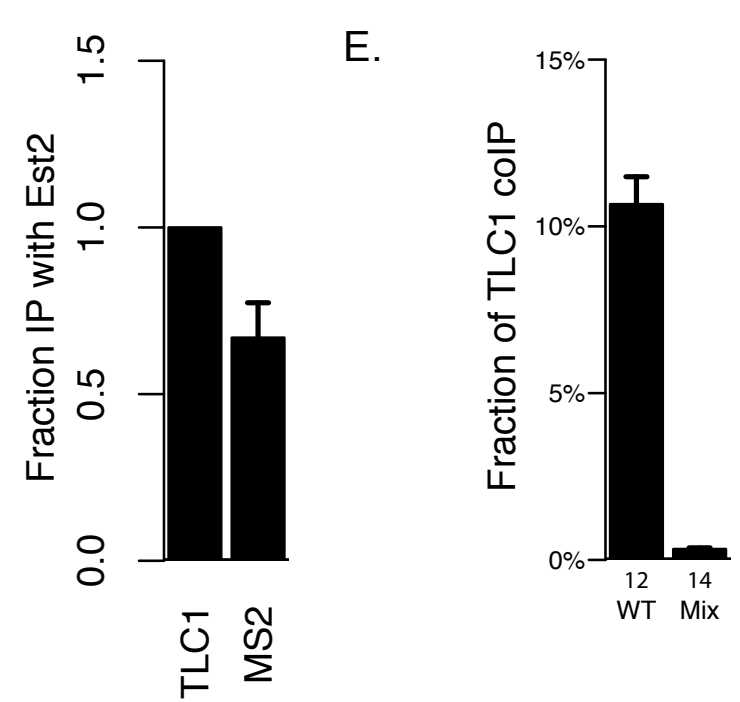

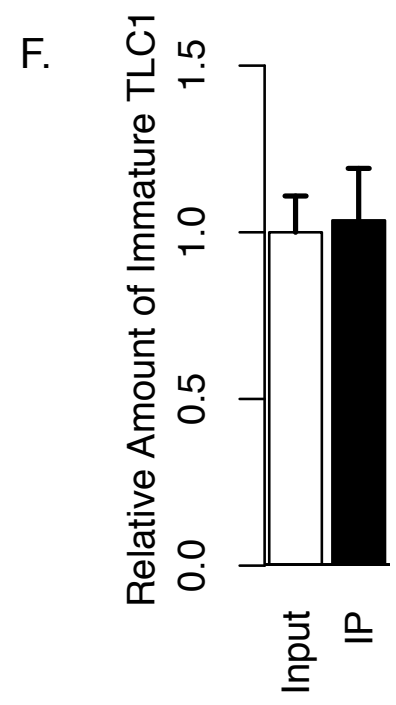

G.

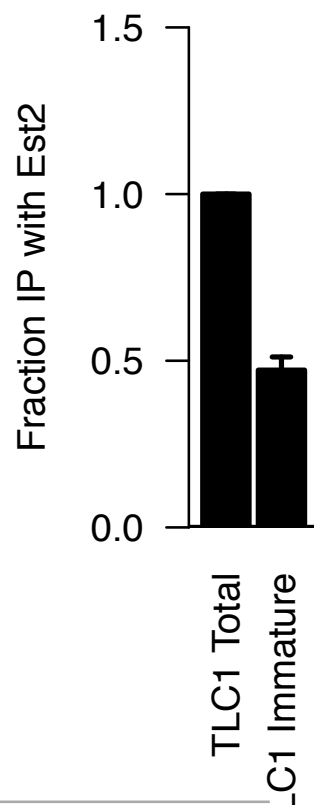




\section{Figure 3 (on next page)}

Regions of TLC1 involved in TLC1-TLClassociation

3A. Anti-sense oligonucleotides can disrupt TLC1-TLClassociation. Anti-sense oligonucleotides were designed against TLC1 and incubated with the MS2 immunoprecipitates. The lysates from yEHB22,807/808 were used for these experiments. The fraction of untagged TLC1 that remained on the beads with the MS2-tagged TLC1 compared to the average of the sample that had no oligonucleotides or random oligonucleotides added is shown. All 72 anti-TLC1 primers or 72 random primers were added. The error bars indicate the standard errors between the two experiments. 3B. Different subsets of oligonucleotides were added during the wash step of immunoprecipitation. The lysates from yEHB22,807/808 were used for these experiments. Each box represents the TLC1 region against which oligonucleotides added targeted. Each ninth and third region contained 8 and 24 oligonucleotides respectively. Shown in each box is the fraction of TLC1 that remained on the beads after the competition assay compared to the average of the sample that had no oligonucleotides or random oligonucleotides added (standard deviation in parentheses). 3C. The TLC1 3' region that is cleaved off plays a role in TLC1 dimerization. The fractions of TLC1 in dimer form, normalized to WT, are shown for strains that carry mutations that disrupt palindromic sequence in the 3' region of TLC1. WT=CGCGCG (yEHB22,662/663, yEHB22,807/808), 42G=CGGGGG (yEHB22,742/743, yEHB22,809/810), 42C=CCCCCG (yEHB22,744/745, yEHB22,811/812), 42G+C=CGGGGG+CCCCCG (yEHB22,776/777, yEHB22,813/814). The error bars indicate the standard errors among the samples. The numbers of experiments represented are shown just below the $\mathrm{x}$-axis. 
Figure 3

A.

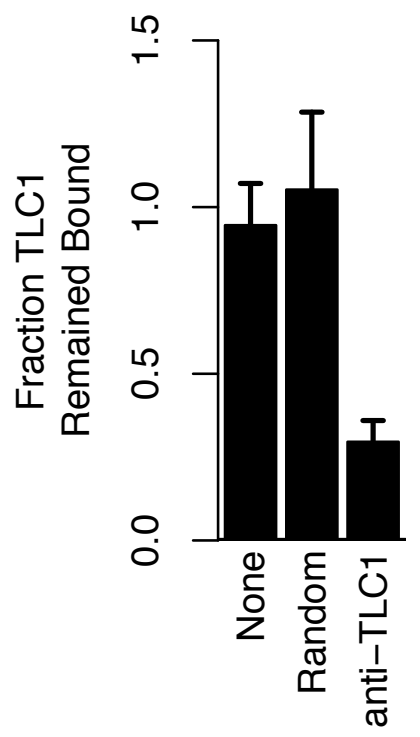

C.

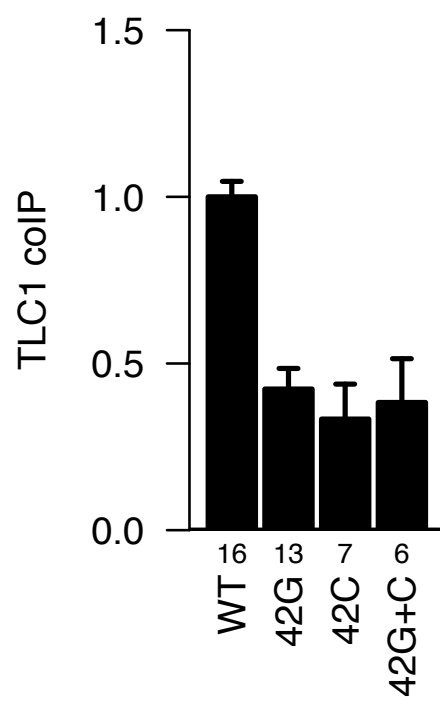

B.

TLC1 Regions

\begin{tabular}{|c|c|c|c|c|c|c|c|c|c|c|}
\hline from $\mathrm{Fl}$ & gure $1 \mathrm{~A}$ & 1 & 2 & 3 & 4 & 5 & 6 & 7 & 8 & 9 \\
\hline \multirow{3}{*}{$\begin{array}{c}0.95 \\
(0.13)\end{array}$} & \multirow{3}{*}{$\begin{array}{c}1.05 \\
(0.23)\end{array}$} & $\begin{array}{c}1.16 \\
(0.19)\end{array}$ & $\begin{array}{c}1.06 \\
(0.06)\end{array}$ & $\begin{array}{c}1.18 \\
(0.25)\end{array}$ & $\begin{array}{c}1.06 \\
(0.03)\end{array}$ & $\begin{array}{c}1.13 \\
(0.09)\end{array}$ & $\begin{array}{c}0.88 \\
(0.06)\end{array}$ & $\begin{array}{c}0.87 \\
(0.02)\end{array}$ & $\begin{array}{c}0.99 \\
(0.14)\end{array}$ & $\begin{array}{c}0.70 \\
(0.05)\end{array}$ \\
\hline & & \multicolumn{3}{|c|}{$\begin{array}{c}0.96 \\
(0.15)\end{array}$} & \multicolumn{3}{|c|}{$\begin{array}{c}0.82 \\
(0.15)\end{array}$} & \multicolumn{3}{|c|}{$\begin{array}{c}0.66 \\
(0.03)\end{array}$} \\
\hline & & \multicolumn{9}{|c|}{$\begin{array}{c}0.30 \\
(0.06)\end{array}$} \\
\hline
\end{tabular}

None ${ }^{\text {Random }}$

Oligonucleotide-Targeted Regions

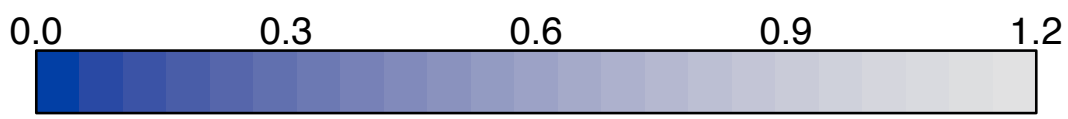

Fraction of Untagged TLC1 Remained Bound After Oligonucleotide Competition 


\section{Figure 4 (on next page)}

\section{TLC1-TLC1 Association and TLC1 Biogenesis}

4A. TLC1 transport to the cytoplasm is required for TLC1-TLC1 association The fractions of TLC1 in dimer form, normalized to WT, are shown for strains that have indicated genes involved in TLC1 biogenesis pathway is deleted. WT $=y E H B 22,662 / 663, \operatorname{tgs} \Delta=$ yEHB22,704/705, nup133D = yEHB22,768/769. The error bars represent standard errors among the samples, and the numbers of experiments represented are shown just below the $x$-axis. The asterisk denotes $p<0.05$. 4B. Cells (yEHB22,662/663) were arrested in alphafactor, released and collected every 15 minutes. The first sample ( $t=0 \mathrm{~min}$ ) is from alphafactor arrested cells. Levels of cyclin mRNAs measured to track cell-cycle progress. The values are normalized so that the lowest value is 0 and the highest value is 1 . The horizontal bars show cell cycle phase ascertained from the measured cyclin mRNA expression levels shown. 4C. Total TLC1 levels, tagged and untagged, are shown, normalized to the asynchronous culture. The error bars indicate standard deviation between two experiments. 4D The fraction of TLC1 in dimer form calculated from colP experiments are shown. The values are normalized to the asynchronous sample, and the error bars represent the standard deviation between two experiments. 
Figure 4

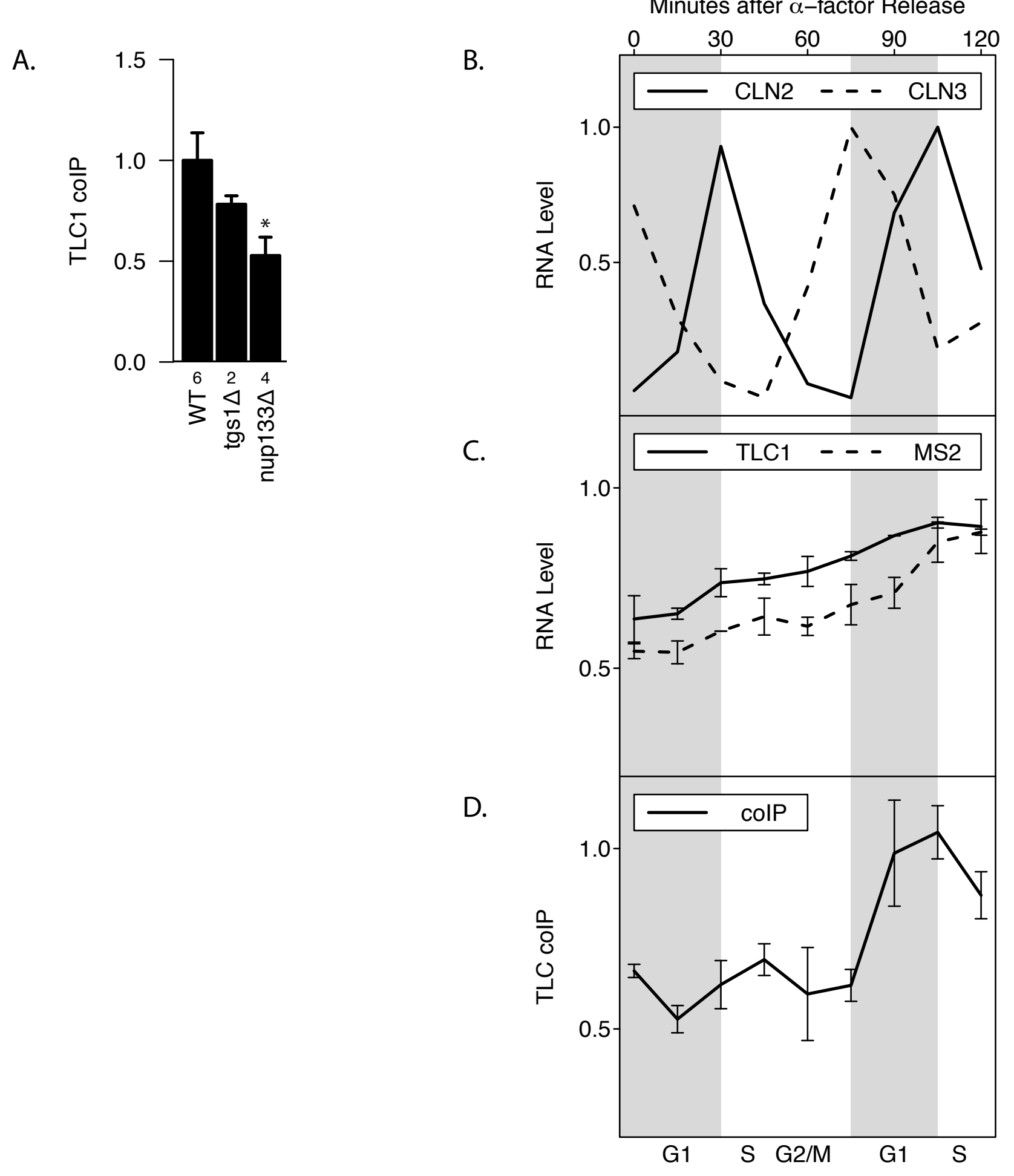

B.

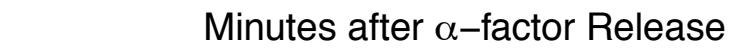




\section{Figure 5 (on next page)}

\section{Protein factor requirements for TLC1-TLC1 association}

5A. TLC1 dimerization is partially sensitive to trypsin treatment. The fraction of TLC1 that remained in the dimer form after mock or trypsin treatment was measured in lysates from WT strains (yEHB22,807/808). The values were normalized to the average of trypsinuntreated samples. The error bars indicate the standard error between two experiments. The numbers of experiments represented are shown just below the x-axis. 5B. TLC1 dimerization is only modestly affected by absence of Est 1, 2 or 3 . The fractions of TLC1 in dimer form, normalized to WT, are shown for strains that have indicated gene is deleted. WT = yEHB22,662/663, est1 $\Delta=y E H B 22,698 / 699$, est2 $\Delta=y E H B 22,724 / 725$, est3D $=$ yEHB22,700/701. The error bars represent the standard deviation between samples. The numbers of experiments represented are shown just below the $x$-axis. The asterisk denotes $p$ $<0.05$. 5C. Est2 interactions with TLC1 affect TLC1-TLC1 associations. The RNA pseudoknot structure critical for Est2 binding to TLC1 was mutated (cs3 and cs4) and compensatory mutation (cs3-cs4) was introduced. The fractions of TLC1 in dimer form, normalized to WT, are shown for strains carrying these tlc1 mutations. The strains yEHB22,722/723 carrying CEN-ARS plasmids were used: WT = pRS316-TLC1, cs3 = pRS316-tlc1-21, cs4 = pRS316-tlc120, cs3-cs4 = pRS316-tcl1-22. These plasmids were generous gift from Jue Lin (Lin et al., 2004). The error bars indicate the standard error, and the numbers of experiments represented are shown just below the $\mathrm{x}$-axis. 5D TLC1 dimerization requires $\mathrm{Ku}$. The fractions of TLC1 in dimer form, normalized to WT, are shown for strains mutated for indicated genes. $\mathrm{WT}=\mathrm{yEHB22,662/663}, \mathrm{yEHB22,750/751,yEHB22,807/808,yku70D}=\mathrm{yEHB22,682/683}$, yku80 $\Delta=$ yEHB22,662/663, yku80-135i $=$ yEHB22,750/751, yEHB22,815/816, tel1 $\Delta=$

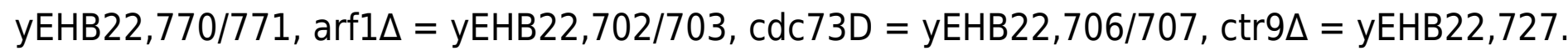
The error bars indicate the standard error among the samples, except for ctr9 sample, which was done only once. The numbers of experiments represented are shown just below

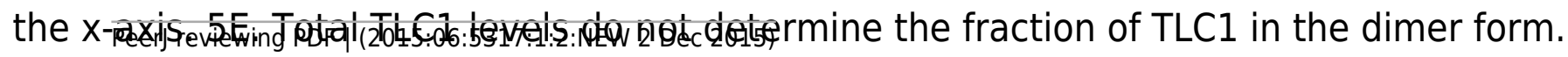


Untagged TLC1 levels in the total RNA were measured in strains deleted for the indicated genes. The levels were normalized to PGK1 mRNA levels first and then to the wild-type levels. The error bars indicate the standard error among the samples, except for $\operatorname{ctr} 9 \Delta$ sample, which was done only once. The strains used are the same as noted in Figure 4D. The numbers of experiments represented are shown just below the x-axis. 
A.

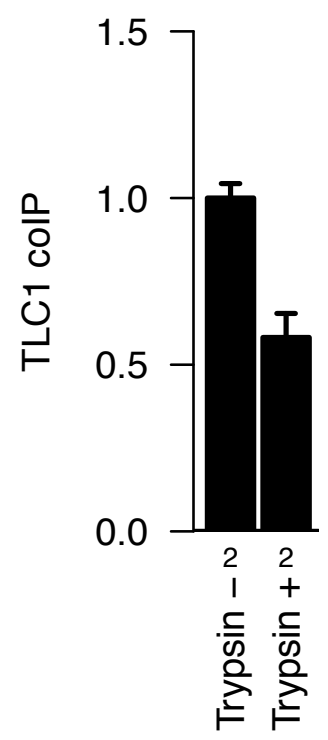

B.

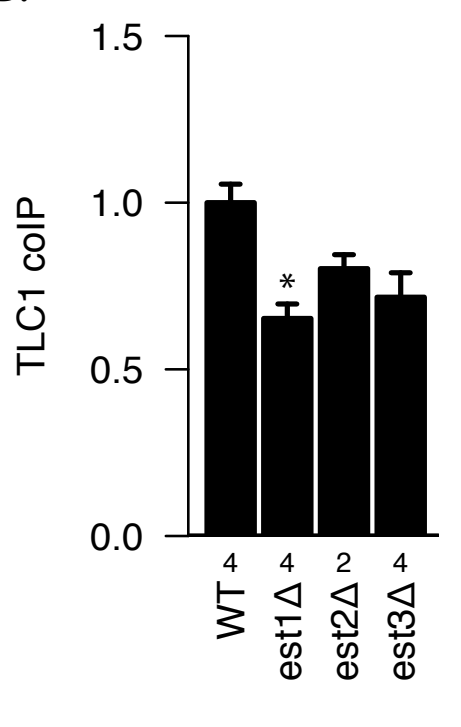

C.

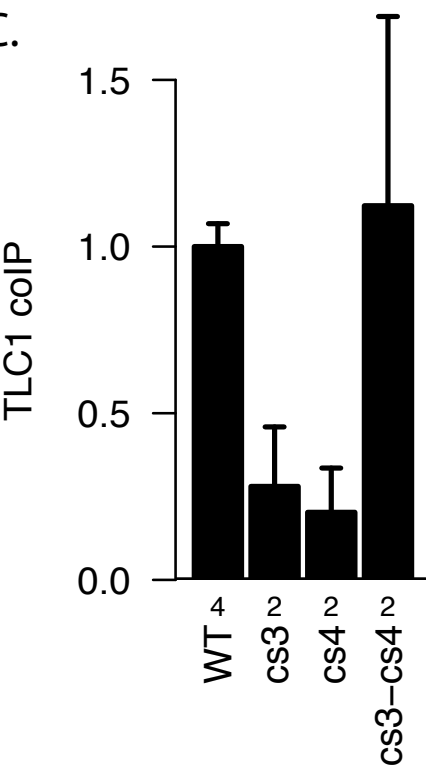

D.

E.
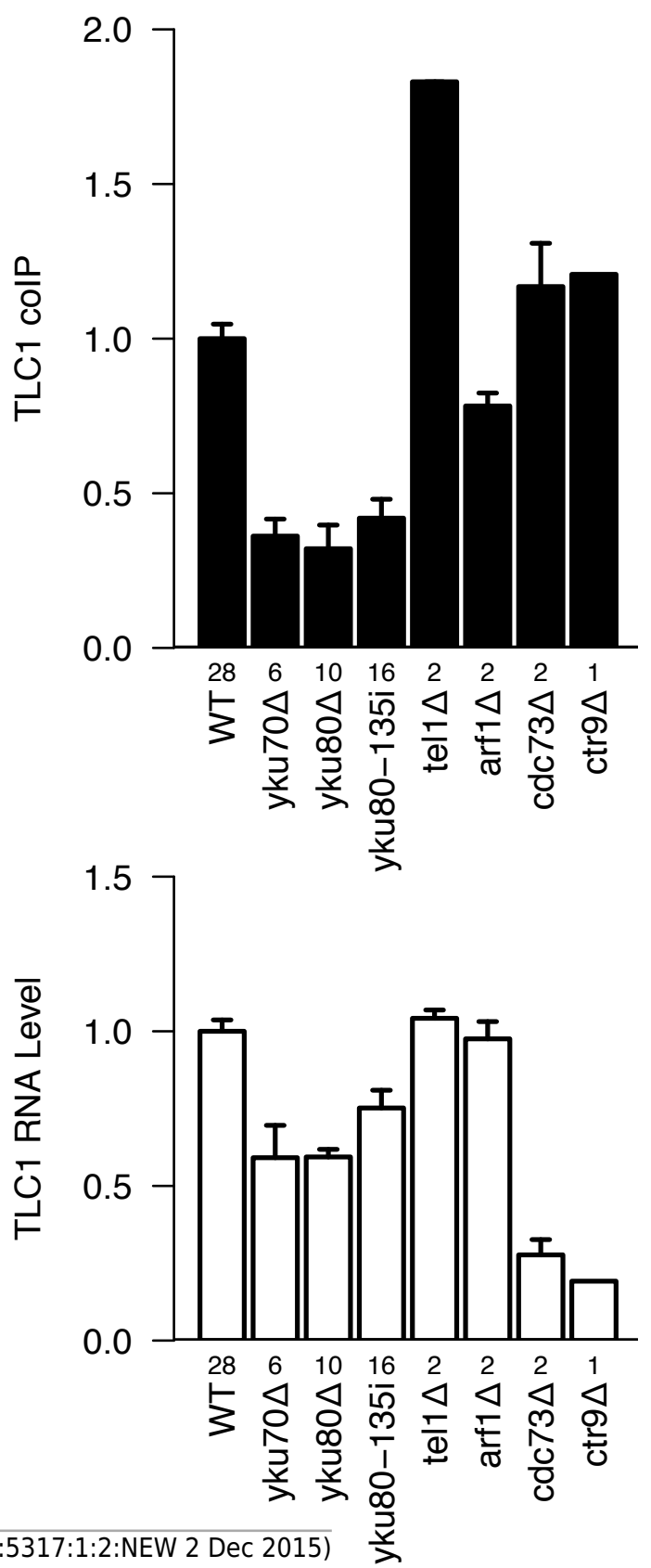


\section{Figure 6 (on next page)}

Two separate pathways of TLC1-TLC1 association

6A. Ku complex binding to TLC1 and Sir4 are required for TLC1-TLC1 association but telomere tethering to the nuclear periphery and telomere silencing are not. Mutations defective in either telomere tethering to nuclear periphery $(c t f 18 \Delta=\mathrm{yEHB22,764/765}$, and esc1D $=$ yEHB22,766/767) or telomere silencing (sir2 $\Delta=y E H B 22,728 / 729, y E H B 22,819 / 820, \operatorname{sir} 3 D=$ yEHB22,762/763, sir4 $\Delta=y E H B 22,730 / 731, y E H B 22,817 / 818$, and sir4-42 $=y E H B 22,787 / 788$ ) are indicated. The fractions of TLC1 in dimer form, normalized to WT (yEHB22,662/663, yEHB22,807/808), are shown for strains indicated. The error bars indicate the standard error, and the numbers of experiments represented are shown just below the $\mathrm{x}$-axis. 6B. Lysate was either untreated, mock-treated, or treated with DNase prior to immunoprecipitation. The efficient loss of DNA was validated only in DNase treated samples. Despite the loss of DNA in the samples, the TLC1-TLC1 colP efficiency was not reduced. WT strains (yEHB22,662/663) were used for "Coex" samples. In "Mix" samples, strains expressing tagged and untagged TLC1 independently (yEHB22,720/721 and yEHB22,722/723) were mixed before the lysis step. The fraction of TLC1 in dimer form, normalized to the average of "Coex" samples are shown. The error bars indicate the standard error, and the numbers of experiments represented are shown just below the x-axis. 6C. The Ku mutations were combined with SIR4 deletion. The values are normalized to the average of the wild-type samples in each experiment. The error bars indicate the standard deviation among the samples. The fractions of TLC1 in dimer form, normalized to WT, are shown for strains indicated. WT = yEHB22,662/663, yEHB22,807/808, sir4 $\Delta=$ yEHB22,730/731, yEHB22,817/818, yku80-135i = yEHB22,750/751, yEHB22,815/816, yku80-135i sir4 $\Delta=y E H B 22,821 / 822, y k u 80 D=$ yEHB22,662/663, yku80 $\Delta$ sir4 $\Delta=y E H B 22,774 / 775$. The error bars indicate the standard error, and the numbers of experiments represented are shown just below the x-axis. 6D. The $\mathrm{Ku}$ and Sir4 combined with the mutation in the $3^{\prime}$ region. The deletion strains for SIR4 and YKU8Q 
normalized to WT, are shown for strains indicated. WT = yEHB22,662/663, yEHB22,807/808, $42 \mathrm{G}=\mathrm{yEHB22}, 742 / 743, \mathrm{yEHB} 22,809 / 180, \operatorname{sir} 4 \Delta=\mathrm{yEHB22}, 730 / 731, \mathrm{yEHB} 22,817 / 818$, sir4D $42 G=y E H B 22,823 / 284, y k u 80 \Delta=y E H B 22,662 / 663, y k u 80 \Delta 42 G=y E H B 22,776 / 777$. The error bars indicate the standard error, and the numbers of experiments represented are shown just below the $x$-axis. 
Figure 6

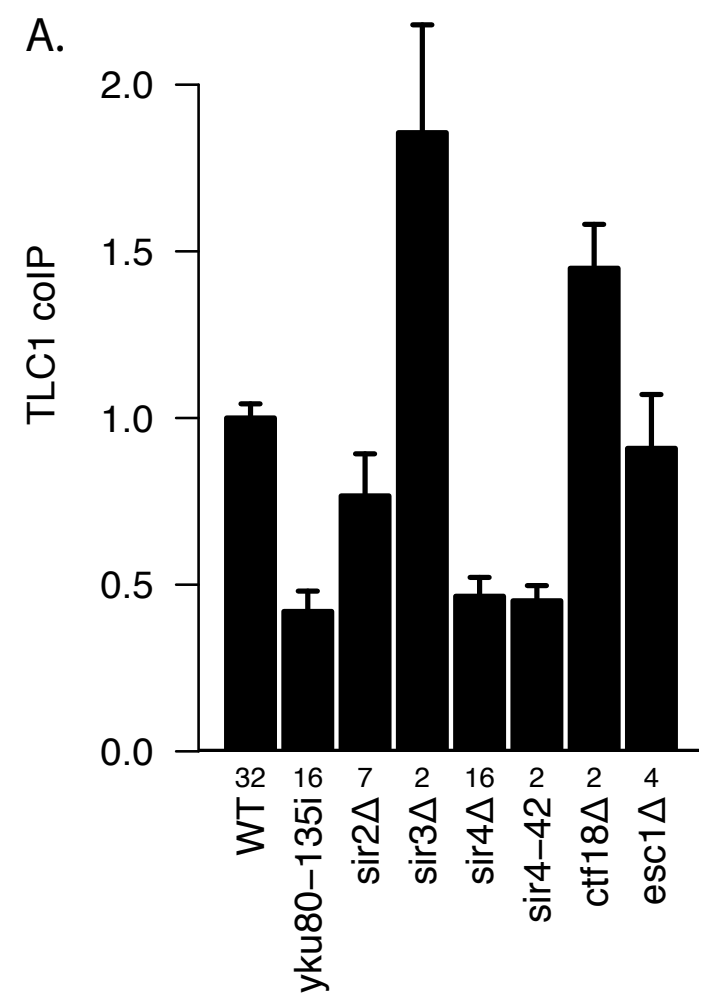

B.

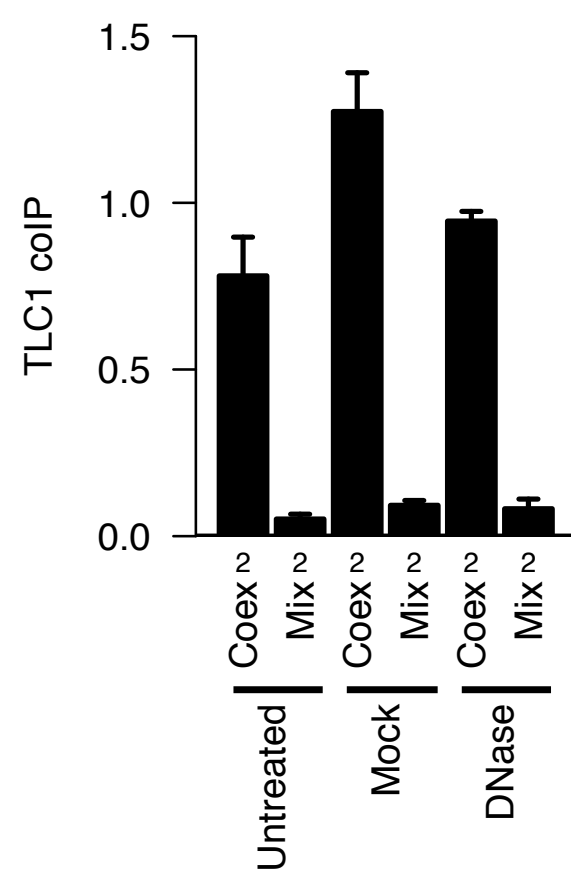

C.

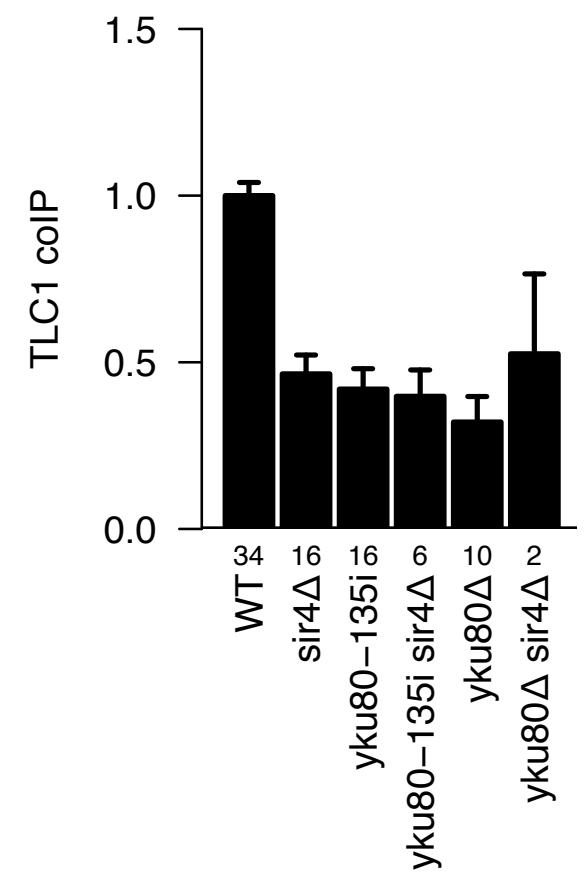

D.

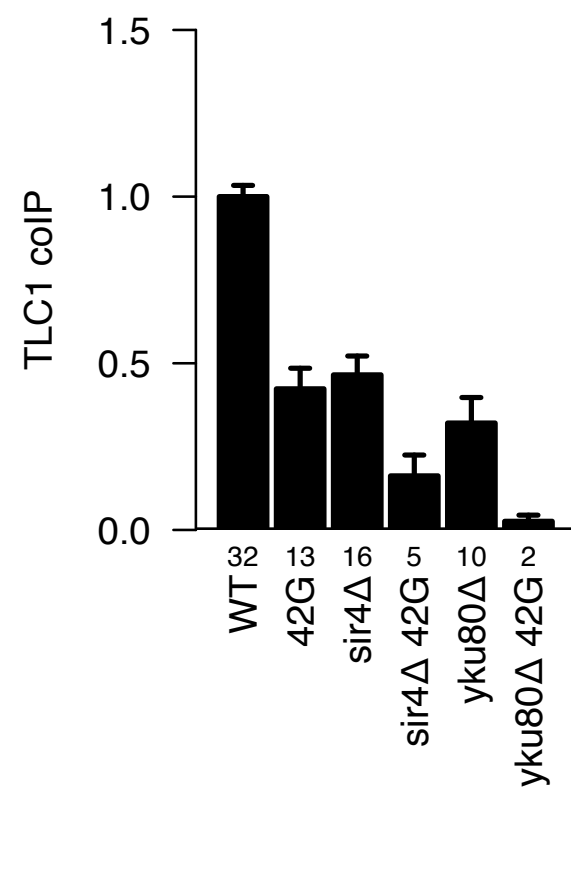




\section{Figure 7 (on next page)}

Lack of Evidence for Est2-Est2 association in vivo

7A. Est2- $\triangle C P$ lacks the Est2 TLC1 binding domain. Myc-tagged Est2 (either Est2-Myc or Est2$\Delta \mathrm{CP}$ ) was immunoprecipitated from lysates of strains co-expressing Est2-HA. Fractions of

TLC1 isolated are shown. Error bars represent standard deviation from two experiments. The table below identify whether CP region of Est2 is deleted $(\Delta)$ in Myc or HA-tagged Est2. The strains used in these experiments were generous gift of Jue Lin (Lin \& Blackburn, 2004) . 7B. The amount of TLC1 immunoprecipitated after sequential immunoprecipitation, anti-FLAG then anti-MYC, was measured. Amount of TLC1 remained in the MYC IP is represented as the fraction of TLC1 immunoprecipitated in the FLAG IP. The table below indicates EST2 fusions with specified tags present in each IP. The strains yEHB22,825-827 were used in these experiments. 
A.

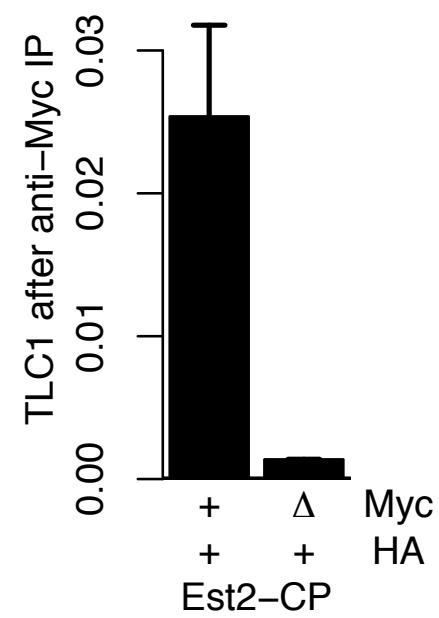

B.

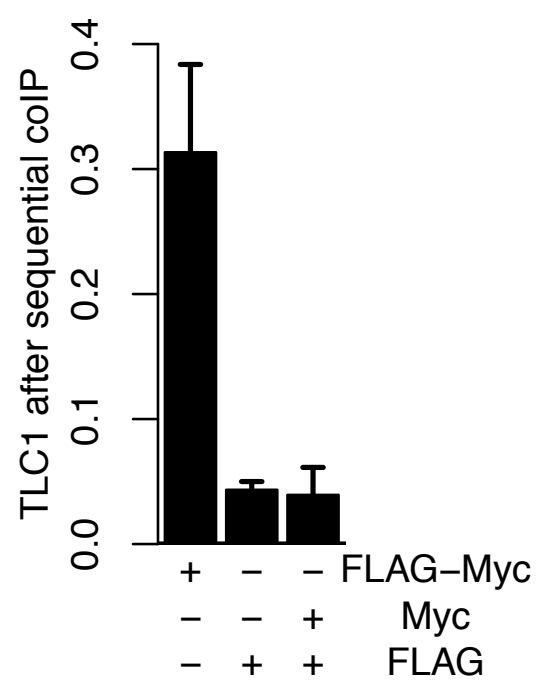

Est2 Fusion Tags 


\section{Figure 8 (on next page)}

Two modes of dimerization model

Top: Schematic of TLC1 cleavage of 3' region. Tick marks: template region of TLC1. CGCGCG: sequence at the 3' region important in TLC1 dimerization. The stem-loop structure that the Ku complex binds is indicated. (Middle) Two modes of TLC1-TLC1 association in vivo. Mode I, dependent on the precursor TLC1 3' region, is initiated before the $3^{\prime}$ region is cleaved off. Mode II, dependent on Sir4/2 and the Ku complex, possibly at telomeres. TLC1 in the telomerase RNP is either monomeric or dimeric, but each RNP contains only one Est2 (bottom). 

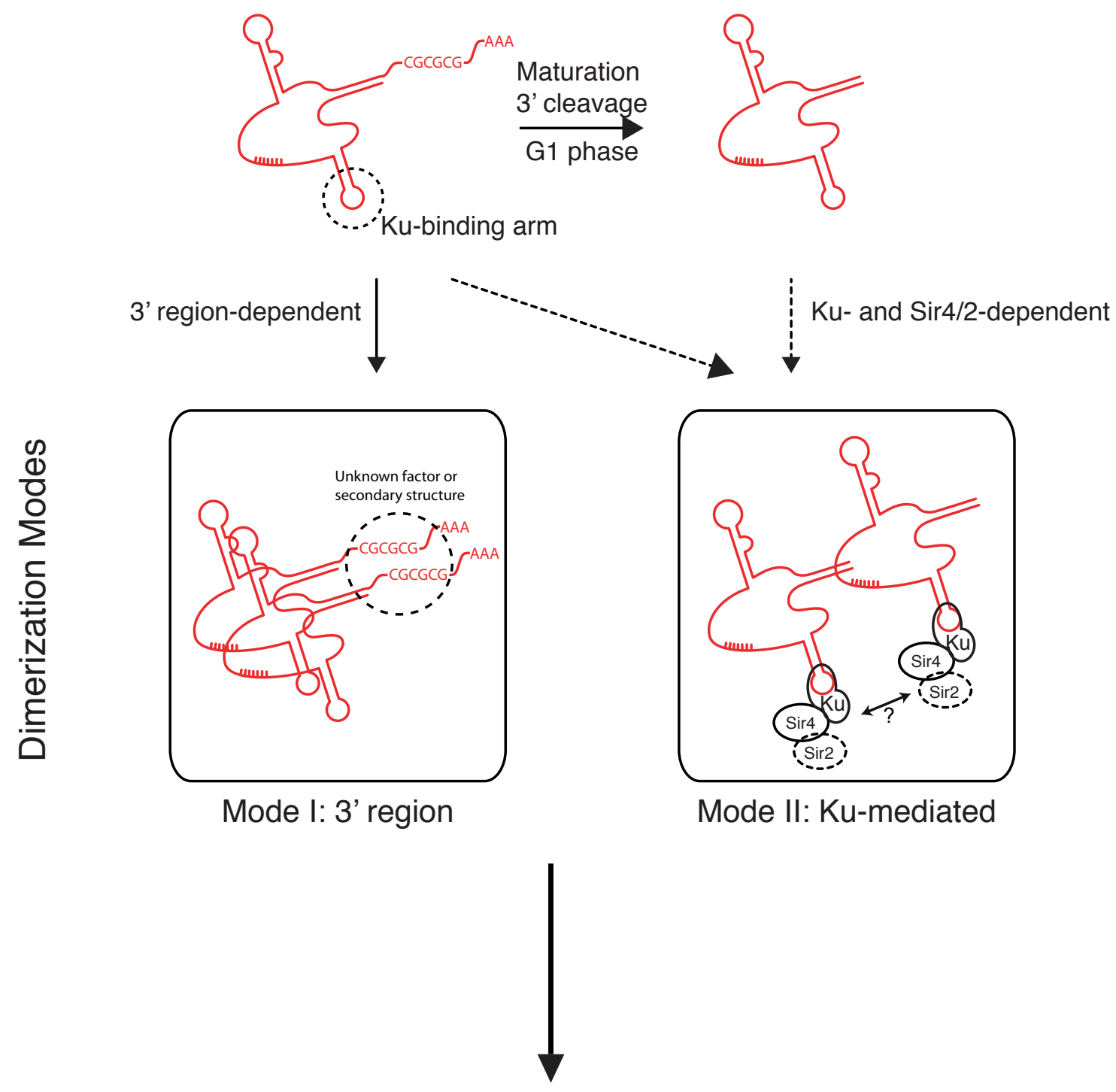

Single Reverse Transcriptase (Est2)

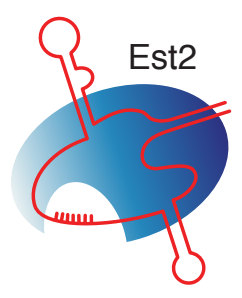

+ one copy of TLC1
$\mathrm{OR}$

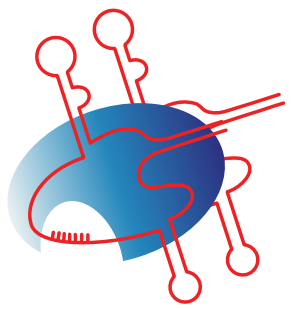

+ two copies of TLC1 